\title{
Du crayon au stylo numérique : influences des IHM à stylo et des interprétations numériques sur l'activité graphique en tâches de conception
}

\author{
STEPHANE SAFIN \\ ROLAND JUCHMES \\ PIERRE LECLERCQ \\ LUCID-ULg - Lab for User Cognition and Innovative Design - Université de Liège
}

\begin{abstract}
Résumé : Notre recherche s'inscrit dans le cadre du développement de logiciels de support à la conception basés sur le dessin à main levée dans le domaine de l'architecture. Dans cet article, nous étudions l'activité de duplication de dessins observée lors des changements de calques en phase d'esquisse préliminaire. Nous cherchons à identifier les impacts de deux environnements logiciels d'esquisses numériques, avec ou sans interprétation du dessin, sur cette activité ainsi que sur les productions graphiques. Nous observons six activités de conception, deux sur papiercrayon et quatre dans deux environnements numériques, et déduisons certaines conclusions opérationnelles pour le développement de logiciels destinés à supporter le croquis d'architecture en phase de conception préliminaire
\end{abstract}

Mots clés : Conception architecturale, esquisses numériques, interprétation de croquis.

\begin{abstract}
Our research is embedded in the framework of the development of free-hand drawing-based computer assisted architectural design environment. In this paper, we study the activity of drawings duplication, observed during the paper changes in the phase of preliminary sketching. We aim at identifying the impacts of two digital sketches environments, with or without drawings interpretation, on this activity and on the graphical productions. We observe six activities, two with paper-pencil and four distributed on our two prototypes, and draw some operational conclusions for the development of software designed to support the architectural sketches in the preliminary design stage.
\end{abstract}

Key words: Architectural design, digital sketches, sketches interpretation.

Les articles de JIPS sont publiés sous licence Creative Commons Paternité 2.0 Générique. 


\section{INTRODUCTION}

Ce travail s'inscrit dans un large contexte d'études d'usages pour le développement d'applications de conception assistée par ordinateur sur base d'esquisses. Ce type d'environnement pose un certain nombre de questions sur la manière dont les activités d'expression graphique sont modifiées par les atouts et limitations de tels systèmes. En effet, l'activité de pré-conception en architecture est caractérisée par une succession de croquis et d'esquisses qui, à mesure qu'elles se succèdent, permettent de préciser la pensée de l'architecte et l'objet de sa conception [Lebahar, 1983]. Malgré la prolifération des outils de CAO parfois très performants, l'esquisse papier-crayon, par la souplesse qu'elle autorise, reste le moyen privilégié d'expression dans ces phases préliminaires. Mais la transposition de l'esquisse papier en esquisse numérique pose légitimement la question de l'impact de cette numérisation des supports. En particulier, nous nous intéressons dans cet article aux modifications induites par un environnement d'esquisses numériques dans l'activité de remise au net de croquis, ainsi que sur les caractéristiques du croquis lui-même.

Cette étude comprend plusieurs objectifs :

- identifier l'impact d'un environnement numérique sur les caractéristiques graphiques des dessins d'architecture en phase préliminaire de conception ;

- identifier les modalités et fonctions de successions des esquisses, en adoptant une perspective dynamique ;

- identifier les comportements et activités cognitives de ce qui est appelé habituellement la « mise au net de dessins ».

Notre recherche prend comme point de départ un constat réalisé lors d'une étude préalable [Safin, Juchmes \& Leclercq 2007] mettant en lumière une différenciation fonctionnelle et graphique entre des dessins «nets » et des dessins « de brouillon » dans les activités de conception architecturale. Alors que ces deux types de dessins ont été identifiés aussi bien dans un environnement numérique que sur papier-crayon, les modalités de leur construction n'ont pas été étudiées en détail. Notre objectif est d'avancer dans la compréhension de la dynamique de cette construction graphique afin d'orienter le développement de nouveaux outils basés sur l'esquisse (et notamment la re-conception de l'un de nos précédents prototypes).

Afin d'appréhender l'activité de mise au net de dessins et l'influence d'un environnement de dessin numérique sur cette activité, nous dressons dans un premier temps un court état de l'art sur les interfaces-esquisses, nous explorons ensuite la notion de transformation des croquis dans la littérature, puis adoptons une démarche empirique visant à identifier les influences des environnements de croquis numériques dans les activités visées. Enfin, nous interprétons nos observations au regard des possibilités et contraintes des systèmes technologiques, en vue de proposer des recommandations pour la conception et le développement de logiciels de support à la préconception architecturale sur base de croquis numériques.

\section{CARACTERISTIQUES DES ESQUISSES ARCHITECTURALES}

La réalisation d'un projet architectural comprend de nombreuses étapes, fait appel à différents acteurs et utilise des ressources et représentations variées [Prost 1992]. Lors des premières phases du projet, la plupart des concepteurs continuent d'utiliser l'esquisse 
réalisée sur papier plutôt que les systèmes informatiques d'assistance au dessin [Bilda et Damakian 2002]. L'esquisse de conception à main levée présente en effet des caractéristiques graphiques bien adaptées aux activités créatives caractéristiques de ces premières étapes: rapidité d'exécution et expressivité graphique [Rogers, Green \& McGrown 2000], abstraction et ambiguïté [MacCall, Ekaterini \& Zabel 2001]. Graphiquement, elles sont composées de nombreux traits imprécis, parfois redondants, présentant peu de variétés (types, couleur, épaisseur etc.) À l'inverse, le dessin numérique réalisé à l'aide de logiciels courants de DAO (dessin assisté par ordinateur) se caractérise par une grande variété de traits, une grande précision et peu d'ambiguïté [Leclercq 2007, Leclercq \& Elsen 2007]. Le dessin à main levé est en outre jugé moins fini et plus créatif que le dessin numérique [Brown 2003]. Peu d'études cependant nous renseignent sur les caractéristiques propres au croquis numérique, constituant l'objet de cette étude.

\section{OUTILS D'INTERPRETATION D'ESQUISSES ARCHITECTURALES}

De nombreux auteurs ont proposé des outils tentant d'exploiter les esquisses architecturales dans un environnement numérique. Nous distinguons trois approches.

- Les environnements numériques basés sur l'analogie avec les outils traditionnels. Ces environnements ne permettent pas une interprétation des esquisses, mais proposent une transposition dans un environnement informatique des outils traditionnellement utilisés en conception architecturale préliminaire: papier, crayons, gomme, feutres, calques etc. Ils peuvent donc être comparés aux logiciels de dessin utilisés par les graphistes. Nous pouvons par exemple citer «transluscent patches » [Kramer 1994] qui est basé sur l'utilisation de la transparence et une métaphore du calque ou encore Electronic Paper [Aliakseu, Martens \& Rauterberg 2006] qui est un système multimodal alliant geste et dessin pour supporter les activités de conception.

- Les outils d'interprétation de croquis 2D en plan. Ces outils analysent puis interprètent les dessins à main levée pour créer un modèle informatique du projet. The Electronic Cocktail Napkin [Gross 1994] par exemple interprète les diagrammes pour accéder à différentes applications comme la recherche en base de cas. Structural Sketcher [Pranovich, Achten \& Wijk 2002] permet à l'architecte de développer son projet en 2D à partir «d'unités graphiques » du type grilles, contours, circulations etc. Ces systèmes présentent dans leur fonctionnement, de nombreux points communs avec les outils de prototypage rapide d'interface basés sur le dessin à main levée comme SILK [Landay 1996] ou SketchiXML [Coyette 2006]. Sesame [Oh 2006] combine une interprétation des croquis en plan avec des opérations de modélisation plus traditionnelles comme l'extrusion.

- Les outils de modélisation 3D adaptés à la conception préliminaire en architecture. Plusieurs auteurs ont développé des systèmes proposant une alternative aux logiciels de modélisation $3 \mathrm{D}$ basés sur le dessin à main levée. Zeleznik avec le système SKETCH [Zeleznik, Herndon \& Hughes 1996] ou encore [Jatupoj 2005] avec Sketchboard proposent de créer des scènes $3 \mathrm{D}$ par commandes gestuelles au stylo. SmartPaper [Shesh 2004] combine gestes de commandes et interprétation de dessins en projection orthogonale. Différents auteurs proposent de reconstruire des volumes 3D à partir de dessins d'architecture en perspective, citons par exemple Huot [Huot, Dumas \& Hégron 2003] ou [Chen 2008]. Hors du domaine de l'architecture, Teddy [Igarashi, Matsuoka \& Tanaka 1999], SmoothSketch [Karpenko 2006] ou encore ILoveSketch [Bae 2008] 
permettent quant à eux de modéliser des formes courbes à partir de traits esquissés dans un espace 3D.

Une quatrième catégorie qui pourrait être distinguée concerne les outils de collaboration supportant le dessin à main levée. De très nombreux systèmes disposent de fonctions de dessin en collaboration distante. Citons par exemple parmi beaucoup d'autres : Scriblink [http://www.scriblink.com/], Imagination Cubed [http://www.imagination3.com/], Sketchfu [http://sketchfu.com/] ou encore WhiteBoardMeeting pour Skype [http://www.whiteboardmeeting.com/]. Généralement les fonctions graphiques proposées par ces logiciels sont des services annexes à des systèmes de clavardage, audio ou visioconférences. Ils n'autorisent actuellement pas la gestion de plusieurs documents superposés, l'exploitation de grandes surfaces de travail, les calques transparents ou encore l'import d'images- préexistantes. Ces logiciels sont dès lors peu adaptés à la complexité inhérente à l'activité de conception architecturale.

\section{TRANSFORMATIONS DES ESQUISSES ARCHITECTURALES}

Différents auteurs ont étudié la conception préliminaire, notamment en architecture, à travers la succession des représentations graphiques générées. Les esquisses à main levée, largement majoritaires à ce stade du projet, y sont étudiées comme les instantanés caractéristiques de l'évolution du processus de conception. Le processus peut alors être envisagé comme une succession des transformations entre les croquis. C'est l'étude de ces transformations qui nous renseigne sur les intentions du concepteur et sur les opérations cognitives mobilisées.

Goel [1995] analyse le processus de conception en définissant trois types de transformations entre les représentations : les transformations latérales (le dessin évolue d'une solution vers une solution légèrement différente), les transformations verticales (le dessin évolue d'une représentation vers une représentation plus détaillée de la même solution) et les duplications (le concepteur passe d'une représentation à une représentation équivalente).

En reprenant la caractérisation des transformations proposée par Goel, Rodgers, Green et McGown [2000] utilisent la notion de complexité comme mesure de l'évolution du projet. Les croquis sont qualifiés sur une échelle de complexité graphique allant de 1 (dessin au trait monochrome sans annotation ni ombrage) à 5 (nombreux traits, usage intensif des ombrages, annotations expliquant les choix de conception).

Neiman, Do et Gross [1999] utilisent l'analyse rétrospective pour étudier le rôle du raisonnement formel dans le processus de conception. Ils suivent ainsi l'évolution de certains objets architecturaux (colonne, escalier) parmi les différents croquis. Les transformations pistées sont d'ordre géométrique : translations, rotations, changements d'échelle, etc.

Visser [Visser 2006] précise les transformations de Goel et distingue cinq actions de transformations.

- Dupliquer : répliquer ou reformuler un dessin.

- Ajouter : ajouter de nouvelles informations au dessin ou modifier de manière limitée la représentation de base.

- Détailler: retravailler différentes parties d'une représentation en représentations plus détaillées.

- Concrétiser : reprendre une représentation de manière plus concrète.

- Modifier : remplacer par une représentation sans la détailler ni la concrétiser. 
- Substituer : remplacer par une représentation alternative, ni plus détaillée ni concrétisée.

Notre recherche participe à cette approche de caractérisation des transformations entre croquis successifs en se focalisant sur l'activité graphique plutôt que sur le contenu sémantique des dessins. Nous envisageons ainsi non seulement la succession des dessins, mais aussi la dynamique de cette succession. Pour ce faire, nous nous intéressons ici à une activité particulière, présente dans toutes nos observations, généralement qualifiée de « mise au net », consistant à reproduire un croquis de manière plus «soignée». Nous recherchons les caractéristiques de cette activité et ses implications pour le développement d'un environnement supportant l'activité de conception basée sur le dessin d'esquisses.

\section{OBJECTIFS DE L'ETUDE}

Compte tenu de la multiplication des dispositifs d'interfaces-croquis dans les métiers de la conception, de la diversité des points de vue sur l'interprétation de croquis en architecture et en vue de développer des prototypes de soutien à la préconception architecturale, il nous apparaît essentiel d'identifier les influences d'un environnement numérique sur l'activité de dessin. En particulier, nous adressons ici trois classes de questions.

1. Nous cherchons à savoir comment les caractéristiques intrinsèques du dessin à main levée sont modifiées lors de la transposition dans un environnement numérique. Les environnements informatiques de dessin permettent-ils la même richesse d'expression que les outils traditionnels? Les contenus des croquis sont-ils fondamentalement différents dans ces environnements? La façon dont les différents dessins sont répartis sur les supports - papiers réels ou feuilles virtuelles - est-elle dictée ou influencée par l'outil?

2. Nous cherchons aussi à identifier l'influence du dispositif technologique sur le processus de pré-conception architecturale : en quoi l'environnement numérique transforme-t-il la façon dont l'activité de dessin est structurée ? En quoi l'utilisation de calques virtuels modifie-t-elle la succession des dessins et leur répartition? L'interprétation automatique de croquis par un logiciel perturbe-t-il le déroulement de l'activité ? Le dialogue hommemachine induit-il des contraintes sur l'organisation de l'activité ?

3. Enfin, nous nous intéressons à l'activité de mise au net de dessins, caractéristique de la conception architecturale : nous nous posons la question des mécanismes sous-tendant le passage d'une représentation de brouillon vers un dessin net. En effet, alors que les deux types de dessins sont observables et comparables dans les environnements numériques de croquis et sur papier, les modalités de construction de ces dessins peuvent être très variables. La construction de dessins nets sur base de dessins de brouillon est-elle différente sur calques numériques et sur papier-crayon? L'explicitation à un logiciel d'interprétation correspond-elle à la mise au net de versions du bâtiment pour soimême? En d'autres mots, l'approche logicielle change-t-elle radicalement la nature des dessins et de leur construction ou soutient-elle l'activité de mise au net telle que présente « naturellement » à l'étape du croquis ?

En particulier, nous cherchons aussi à distinguer les influences propres à l'environnement de croquis numérique de celles qui sont induites par la présence d'une interprétation des dessins par la machine.

\section{METHODOLOGIE}




\section{1. DISPOSITIF EXPERIMENTAL}

Afin de répondre à ces questions, nous avons observé l'activité de six étudiants ingénieurs et architectes en fin de formation dans un exercice de conception architecturale simplifié mais réaliste, calibré pour une durée de quatre heures maximum. Il s'agit de la réalisation d'une école de 240 élèves en milieu rural, avec un certain nombre de contraintes strictes consignées dans un cahier des charges. Cet exercice, quoique assez éloigné de la complexité de la pratique architecturale, est néanmoins représentatif de l'activité de conception architecturale, c'est-à-dire la définition des concepts-clés, de la forme et du fonctionnement d'un bâtiment.

Afin d'identifier les impacts d'un environnement de croquis numériques ainsi que ceux de l'interprétation, les six participants ont été répartis dans trois conditions :

- dans un environnement «naturel », c'est-à-dire avec les outils traditionnels de dessins : papier, crayon, surligneurs, etc. ;

- dans un environnement d'esquisses numériques de type «palette graphique», sans interprétation logicielle (logiciel SketSha - voir section suivante);

- à l'aide d'un logiciel d'interprétation de croquis numérique d'architecture (logiciel EsQUIsE - voir section suivante).

Les deux dernières conditions ont pris place dans un environnement d'interaction au stylo de grande taille (Bureau Virtuel de conception). En outre, chacun des participants était autorisé à utiliser des règles, équerres et calculatrices. Afin de permettre aux observateurs de contextualiser les actions du concepteur, une procédure classique de think aloud a été mise en place. Il était explicitement demandé au concepteur d'exprimer tout haut le flot de sa pensée, spontanément et sans tenter de la structurer. Une procédure d'une demi-heure d'entrainement au think aloud et, le cas échéant, à l'utilisation des logiciels, a pris place la veille de l'expérience. Cet entrainement avait aussi pour but d'harmoniser les connaissances des sujets quant aux environnements utilisés.

Chacune des activités a été intégralement filmée en plusieurs vues, et analysée conjointement par un ergonome (pour les aspects cognitifs) et un architecte (pour les composantes métier). Le plan expérimental est présenté au tableau 1.

\begin{tabular}{|c|c|c|c|}
\hline & \multicolumn{2}{|c|}{ INTERPRETATION LOGICIELLE } \\
\hline & & Sans & Avec \\
\hline \multirow{2}{*}{ 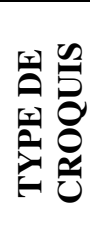 } & Réel & $\begin{array}{l}\text { Papier-crayon } \\
\text { Participant } 1(\mathrm{P} 1) \\
\text { Participant } 2(\mathrm{P} 2)\end{array}$ & $/ / /$ \\
\hline & Numérique & $\begin{array}{l}\text { logiciel SketSha } \\
\text { Participant } 3(\mathrm{P} 3) \\
\text { Participant } 4(\mathrm{P} 4)\end{array}$ & $\begin{array}{l}\text { logiciel EsQUIsE } \\
\text { Participant } 5 \text { (P5) } \\
\text { Participant } 6 \text { (P6) }\end{array}$ \\
\hline
\end{tabular}

Tableau 1 : plan expérimental.

\section{2. OUTILS UTILISES}

La première condition s'est effectuée à l'aide d'outils traditionnels (figure 1) : feuilles de papier, papier calques, crayons, surligneurs, stylos... Aucune consigne particulière n'était donnée aux participants quant aux outils, si ce n'est de circonscrire leur activité de dessin dans un espace déterminé par le champ de la caméra. 


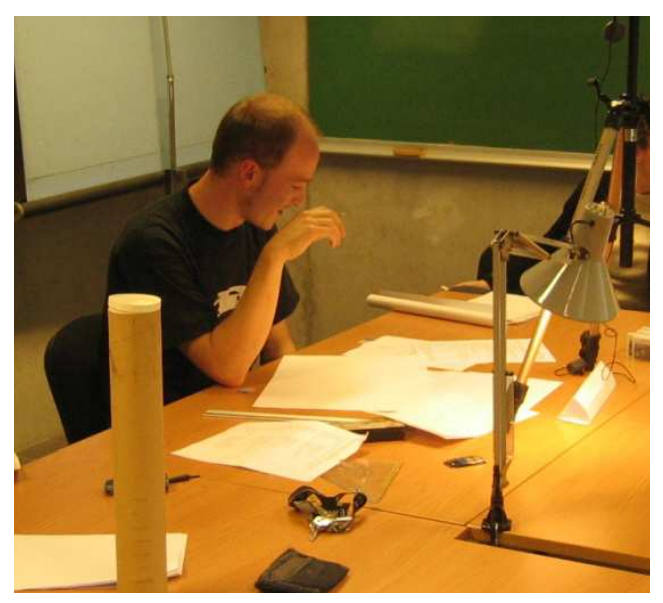

Figure 1 : environnement papier-crayon.

La deuxième condition (environnement numérique sans interprétation) s'est déroulée sur le logiciel SketSha (Sketch Sharing) qui est une palette de dessins partagée (figure 2), développée par le LUCID-ULg.

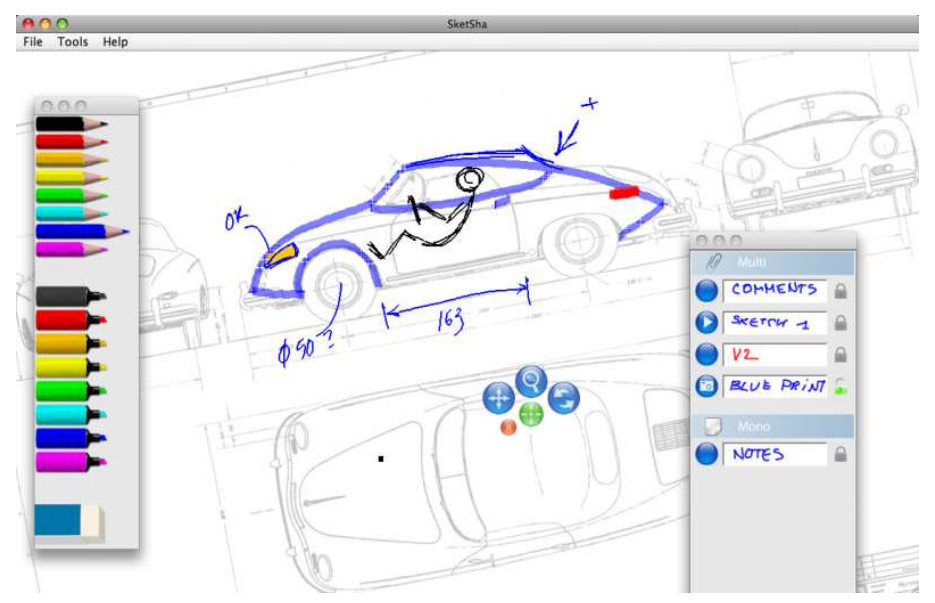

Figure 2 : interface du logiciel SketSha.

Le principe repose sur l'utilisation de plusieurs crayons et surligneurs virtuels dans un système de calques. L'utilisateur peut à sa convenance importer des fonds de plans, créer des calques semi-transparents, les manipuler au stylo à l'aide d'un widget original, les dupliquer et gérer leur ordre de superposition. L'interface se veut la plus épurée possible. Ce logiciel est décrit plus en détails dans Safin, Delfosse \& Leclercq (2010). Si l'intérêt premier de ce logiciel est qu'il permet la collaboration à distance via Internet, pour les besoins de 
l'expérience, le logiciel a été utilisé en mode «standalone » c'est-à-dire en désactivant les fonctions de partage graphique distant.

La troisième condition (dessin numérique avec interprétation) a été effectuée sur le logiciel EsQUIsE, aussi développé par le LUCID-ULg. Ce prototype de recherche, dont le développement est actuellement arrêté, permet à l'utilisateur architecte de dessiner et de recevoir des feedbacks en temps réel, basés sur l'interprétation sémantique de son croquis. Le logiciel EsQUIsE est composé d'un module de saisie graphique et d'un module d'interprétation qui permettent de construire un modèle architectural du bâtiment destiné à alimenter une série d'évaluateurs, notamment une 3D en temps réel (figures 3 et 4).

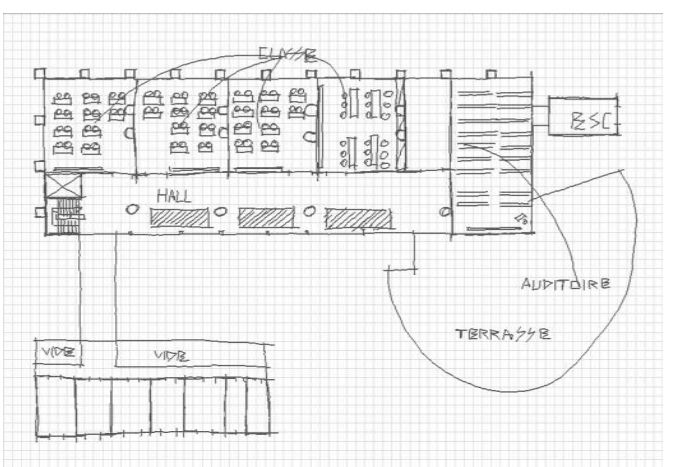

Figure 3. Dessin à main levée sur EsQUIsE.

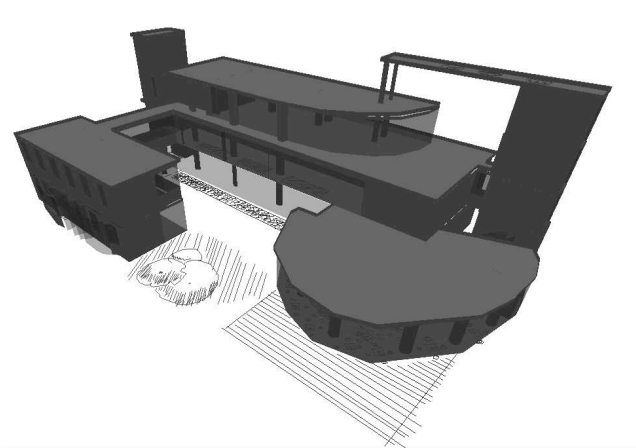

Figure 4 : Modèle 3D auto-généré par EsQUIsE.

L'interface du logiciel (figure 5) est proche de celle de SketSha: une zone large (1) permet le dessin sur calques virtuels à l'aide d'une palette de crayons et d'une gomme numériques (2). Chaque calque est représenté par un onglet (3). L'utilisateur peut librement nommer ces onglets. Il peut aussi les agencer de manière simple (drag and drop) afin de gérer la superposition des calques virtuels dans le modèle. Il peut facilement créer, effacer ou dupliquer des calques grâce aux icônes d'édition (4). Des options de manipulation du calque (rotation, translation, zoom) sont disponibles dans la zone de manipulation (5) et la transparence des calques virtuels est paramétrable. Enfin, la zone des options (6) permet de passer d'un mode à l'autre (mode croquis, mode 3D, autres évaluateurs, préférences...). Le concepteur peut dessiner tout à fait librement sur les calques virtuels grâce aux stylos digitaux de différentes couleurs. Seuls les traits noirs sont interprétés par EsQUIsE pour former le modèle du bâtiment, les autres couleurs servant aux dessins de réflexions et aux annotations. Les calques sont semi-transparents et peuvent être facilement agencés les uns par rapport aux autres.

Notre propos ici n'est pas de détailler le fonctionnement d'EsQUIsE, le développement de ce logiciel étant arrêté. Le lecteur intéressé pourra trouver l'information dans certaines de nos publications précédentes (pour le fonctionnement d'EsQUIsE, voir [Juchmes, Leclercq \& Azar 2004, 2005], pour l'interface, voir [Safin, Boulanger \& Leclercq 2005, Safin et al. 2007]). Ce logiciel étant en cours de réécriture, l'objet de cette étude est de spécifier de nouveaux concepts innovants basés sur les observations issues de l'ancienne version.

En termes de support matériel, ces deux logiciels étaient installés sur le Bureau Virtuel de Conception (figure 6), également produit par le laboratoire LUCID-ULg 


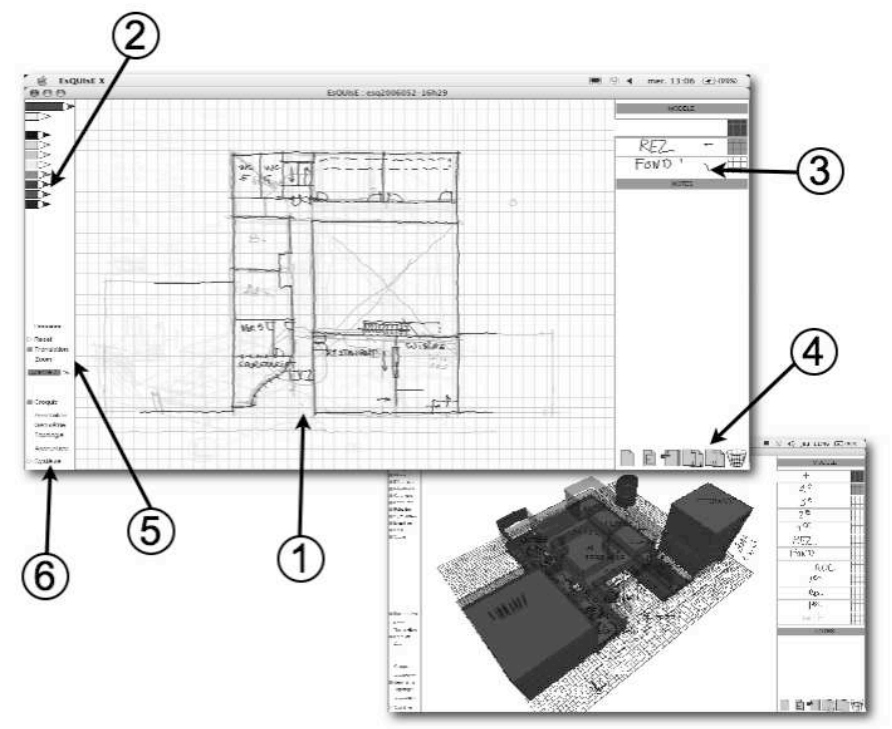

Figure 5. Interface EsQUIsE en mode dessin (image principale) et en mode 3D (seconde image).

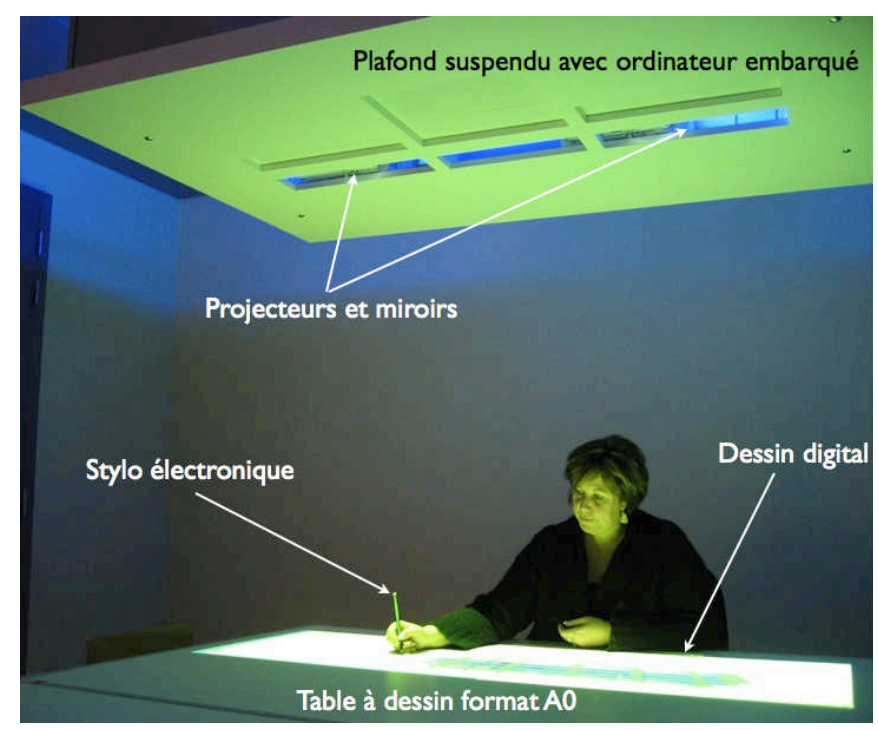

Figure 6 : Bureau Virtuel de Conception.

Ce dispositif se compose d'une table digitale de format A0 s'apparentant à une table à dessin traditionnelle, ainsi qu'un système de projecteurs et miroirs installés dans un plafond suspendu. L'interaction s'effectue à l'aide d'un stylo à solénoïde qui, par son poids et sa forme, s'approche d'un stylo classique. Ce dispositif se veut dans la mouvance de l'ordinateur invisible (Norman, 1998): grâce à ses caractéristiques proches 
d'environnements naturels de travail, l'environnement informatique s'efface de la conscience de l'utilisateur pour qu'il puisse se concentrer sur sa tâche.

EsQUIsE a des points communs avec les trois premières catégories de logiciels d'interprétation d'esquisses telles qu'identifiées dans la section 3 :

- il utilise une analogie avec un carnet de croquis : l'utilisateur peut dessiner avec différents outils numériques (crayons virtuels de différentes couleurs) sur des calques virtuels et gérer la superposition et la transparence de ces feuillets virtuels,

- il réalise une interprétation d'esquisses en plan annotées à main levée,

- il permet la construction en temps réel de la maquette virtuelle du bâtiment et la navigation dans le modèle $3 \mathrm{D}$ généré par le système.

Ce logiciel se positionne ainsi volontairement dans la phase amont du processus de conception.

Par rapport aux mêmes catégories de logiciels d'esquisses identifiées dans la section 3, SketSha possède des points communs avec deux catégories: d'une part il se situe résolument dans la catégorie des outils de collaboration sur base de croquis et d'autre part, comme EsQUIsE, Sketsha utilise l'analogie avec des outils traditionnels, notamment les calques, crayons, surligneurs, etc. Le tableau 2 reprend les principales différences entre les trois environnements.

\begin{tabular}{|l|l|l|l|}
\hline & Situation 1 & Situation 2 & Situation 3 \\
\hline Environnement & Table normale & $\begin{array}{l}\text { Bureau Virtuel } \\
\text { (table électronique) }\end{array}$ & $\begin{array}{l}\text { Bureau Virtuel } \\
\text { (table électronique) }\end{array}$ \\
\hline Logiciel & //I & SketSha & EsQUIsE \\
\hline Outils & $\begin{array}{l}\text { Feutres, stylos, } \\
\text { règles, équerres, }\end{array}$ & $\begin{array}{l}\text { Stylo électronique, } \\
\text { règle }\end{array}$ & $\begin{array}{l}\text { Stylo électronique, } \\
\text { règle }\end{array}$ \\
\hline Feuilles & $\begin{array}{l}\text { Papiers, papiers- } \\
\text { calques }\end{array}$ & Calques virtuels & Calques virtuels \\
\hline $\begin{array}{l}\text { Interprétation des } \\
\text { traits }\end{array}$ & Non & Non & Oui \\
\hline Dessin & Libre & Libre & $\begin{array}{l}\text { Interprété (couleur } \\
\text { noire), libre (autres } \\
\text { couleurs) }\end{array}$ \\
\hline
\end{tabular}

Tableau 2 : Comparaison des trois environnements

\section{3. GRILLES D'ANALYSES}

Les analyses des vidéos des six activités de conception ont porté sur trois volets.

1. D'une part l'analyse des caractéristiques graphiques des dessins. Sur base des productions finales des participants (calques réels ou virtuels), nous avons analysé les propriétés des dessins : nombre de traits, variété, épaisseur, précision...

2. Pour comprendre le processus dans sa dynamique temporelle, nous avons observé l'utilisation des supports de dessins (papiers, papiers-calque et calques virtuels). Partant du principe que les changements de supports ne sont pas anodins en conception, puisqu'ils servent de base aux successions et transformations de dessins, nous avons établi des lignes du temps de l'utilisation de ces différents supports afin d'observer visuellement l'émergence d'une structure dans l'activité de dessin, liée à l'outil. Ainsi, à chaque moment, nous identifions sur quel calque travaille le concepteur et reportons cela 
dans des lignes du temps, arrondi à la minute. Sur ces lignes du temps, nous avons identifié des phases d'activité temporellement circonscrites.

3. Enfin, sur base de cette compréhension temporelle, nous avons sélectionné des moments particuliers de la conception qui sont caractéristiques de l'activité de mise au net, en l'occurrence les moments de création d'un nouveau calque pour le papier-crayon et SketSha, et les phases de travail multi-calques dans EsQUIsE (voir plus loin). Nous avons cherché à comprendre la dynamique du processus de transformation des dessins. Pour ce faire, les extraits vidéo des moments concernés ont été analysés en détail par un ergonome et un architecte. L'objectif est de comprendre le déroulement de ces moments de transformation de l'esquisse du point de vue cognitif mais aussi sémantique et architectural. L'analyse, qui se veut qualitative et exploratoire, vise à définir des concepts d'interaction pour la définition d'interfaces graphiques.

A noter que nous adoptons une démarche qualitative, basée sur une compréhension fine de l'activité.

\section{RESULTATS : LES DIFFERENTS TYPES DE REPRESENTATIONS`}

\section{1. COMPARATIF DES CROQUIS DANS LES DIFFERENTS ENVIRONNEMENTS}

Les croquis observés dans les trois dispositifs expérimentaux présentent des caractéristiques graphiques sensiblement différentes. Nous synthétisons ci-dessous les principales caractéristiques des tracés en fonction de l'environnement de travail dans lequel ils ont été réalisés.

\section{2. CROQUIS SUR PAPIER-CRAYON}

L'activité de dessin à l'aide des outils traditionnels papier-crayon est caractérisée par la production d'une succession d'esquisses ambigües et imprécises, dites de «brouillon» (figure 7). Ces croquis, dessinés exclusivement au crayon, possèdent toutes les caractéristiques des esquisses de conception décrites dans la littérature («thinking sketches » pour Van der Lugt (2005) ou "croquis synthétique» pour Leclercq \& Elsen (2007)) : peu de variabilité et peu de précision dans les traits, incomplétude du dessin, accumulation de traits, vitesse du tracé, coexistences de plusieurs solutions et présence de nombreuses annotations annexes (cotations, notes, calculs...).

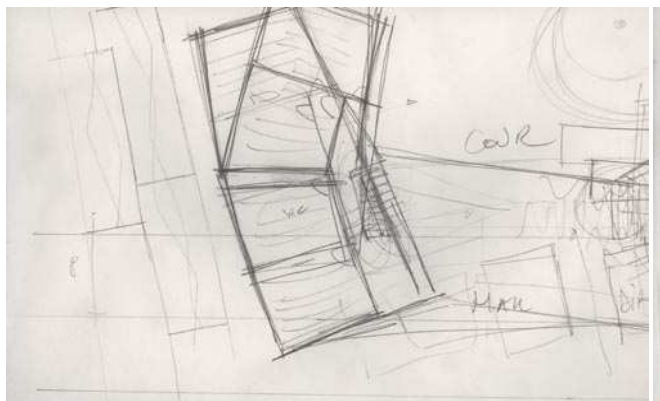

Figure 7 : Esquisse brouillon de P2.

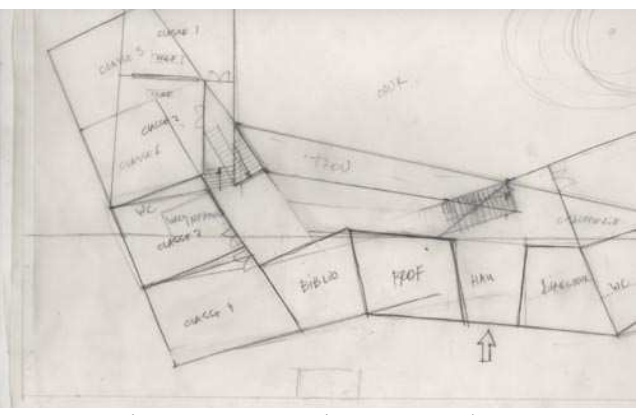

Figure 8 : Esquisse nette de P2. 
Ces esquisses de brouillon sont régulièrement « mises au net » - soit sur un autre calque, soit à même le dessin. A ces moments, le dessin se différencie (figure 8) : les traits ne sont plus accumulés (un trait représente une paroi) et il n'existe plus qu'une solution. Les annotations sont rares et servent uniquement à nommer les espaces. Ces mises au net sont effectuées à l'aide de traits épais de crayons ou au surligneur.

Ces dessins de brouillon et nets, déjà identifiés dans une étude précédente (Safin et al., 2007), sont imbriqués les uns aux autres : les dessins de brouillon fournissent une base aux dessins nets, qui deviennent à leur tour des dessins de brouillon au fur et à mesure des recherches formelles et conceptuelles.

\section{3. CROQUIS SUR SKETSHA}

Sur Sketsha, on observe les mêmes caractéristiques de croquis que dans l'activité papiercrayon. Les deux activités débutent par la production de dessins ambigus et conceptuels (figure 9), voyant s'accumuler de nombreux traits imprécis de couleurs différentes. On observe aussi la présence d'annotations. Au fur et à mesure du déroulement de l'activité, les dessins se font de plus en plus «propres » (figure 10). Peu à peu, le rôle des traits se précise, ils correspondent maintenant clairement à la trace des murs, dans le plan. L'esquisse de simulation laisse progressivement place à une esquisse descriptive du bâtiment et de ses différents espaces. La transparence paramétrable des calques est beaucoup utilisée par le concepteur pour faire coexister ses esquisses. Ainsi, il peut mettre au net ses dessins sur d'autres calques, mais sur le dessin précédent (en paramétrant le calque comme complètement transparent), puis opacifier son calque pour ne voir apparaître que les dessins mis au net.

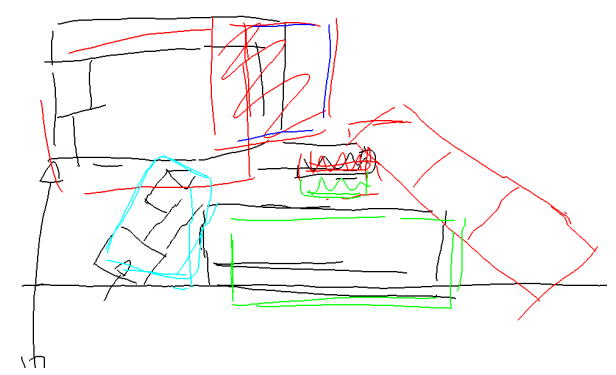

Figure 9 : Esquisse brouillon de P3 (dans les premières étapes)

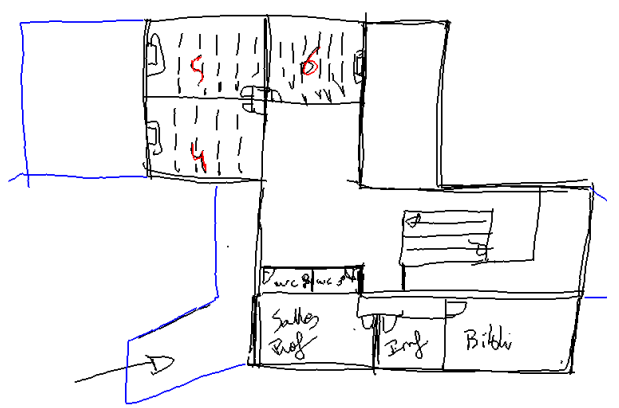

Figure 10 : Esquisse nette de P3 (à la fin du processus)

Au niveau des caractéristiques graphiques des dessins, on remarque quelques différences avec le papier-crayon, induites par l'environnement de conception. D'une part, le dispositif technologique ne permet que deux types de traits (surligneurs ou stylos virtuels). Le nombre total de traits est par conséquent limité, car la multiplication des traits à un même endroit ne modifie pas la saturation des couleurs ni le contraste du trait. D'autre part, pour tirer profit du large espace de travail et compte tenu de l'épaisseur des traits sur l'environnement numérique, l'échelle du dessin est agrandie. 


\section{4. CROQUIS SUR ESQUISE}

Comme pour le papier-crayon, deux types de dessins coexistent. (1) Des dessins de brouillon (figure 11), dessinés exclusivement en couleurs (c'est-à-dire non interprétés par le logiciel) comprenant de nombreux traits, souvent imprécis, se superposant et rarement effacés. De nombreuses solutions et concepts coexistent. De nombreuses annotations et dessins de détails sont présents sur ces dessins. (2) Des esquisses «propres» et mises au net (figure 12), comparables aux dessins nets présents sur papier-crayon: les traits sont plus droits et plus précis que sur les dessins de brouillon, chaque trait représente une paroi et chaque paroi est représentée uniquement par un trait. Ces dessins sont tracés exclusivement en noir (seule couleur interprétée par le logiciel) et le concepteur utilise fréquemment la gomme digitale. A noter qu'en fin de conception, certains de ces dessins nets sont annotés avec des éléments de couleur : aménagements intérieurs, légendes, etc...

Si les dessins sont comparables à ceux observés dans les autres activités, ils se différencient nettement des autres par leur localisation. En effet, on observe très clairement dans les deux activités sur EsQUIsE une spécialisation des calques : les dessins nets et de brouillon sont tracés sur des calques différents, et ne coexistent jamais, comme c'était le cas dans les autres activités. Cette observation est très clairement liée aux propriétés du système : le logiciel, pour fournir au concepteur un modèle 3D cohérent, doit se baser sur des dessins moins ambigus que les esquisses de brouillon. C'est ainsi que tous les traits effectués en noir (seule couleur interprétée par le logiciel) doivent être nets. Or, pour faciliter cette différenciation, il semblerait que les deux concepteurs mettent en place une stratégie de distinction spatiale : il est plus clair pour eux de séparer les esquisses interprétées de celles qui ne doivent pas l'être en les dessinant sur des supports bien distincts.

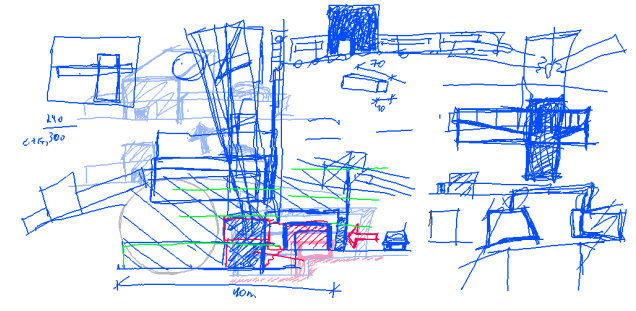

Figure 11 : Esquisse numérique brouillon de P5

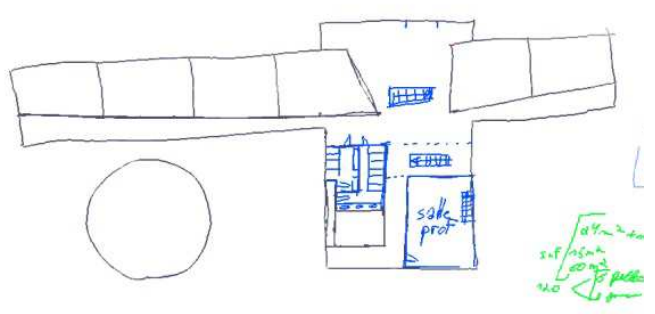

Figure 12 : Esquisse numérique nette de P5

\section{5. COMPARAISONS}

Au point de vue strictement graphique, les dessins observés dans les trois environnements de travail possèdent des points communs et des différences

- L'échelle: les dessins réalisés sur environnement numérique sont plus grands que ceux dessinés sur papier. Cela s'explique par la taille de l'espace de travail - le Bureau Virtuel proposant une surface proche du format A0 alors que les papiers-calque ne sont pas plus grands que le format A3 - et par les caractéristiques des traits - leur épaisseur est fixée à 2 pixels sur l'environnement numérique, taille beaucoup plus importante que la pointe d'un stylo-bille ou d'un crayon bien taillé, et il n'existe que deux types de traits 
(surligneurs ou stylos) alors que le dessin sur papier permet une large palette de subtilités dans le trait (fonction de la pression, de l'inclinaison et du type de crayon). Ainsi, pour pouvoir avoir des dessins comparables, les participants sont dans la nécessité de compenser par une taille supérieure.

- Le nombre de traits: Les dessins sur papier-crayon sont caractérisés par un nombre plus important de traits coexistant sur un même dessin que sur environnement numérique. Cela s'explique aisément par deux raisons : d'une part, comme pour le point précédent, le manque de variabilité du trait rend inutile la superposition de nombreux traits (si deux traits sont parfaitement repassés, il n'en apparaît qu'un seul alors que sur papier-crayon, le trait d'origine devient un peu plus foncé) et, d'autre part, l'utilisation du système de grande taille (Bureau Virtuel) rend sans doute le geste de dessin un peu plus compliqué, incitant l'utilisateur à économiser les traits superflus.

- La quantité d'informations: malgré ces différences importantes des dessins, il s'avère que la quantité d'informations présentes sur les esquisses est relativement similaire dans les différents dispositifs. Ainsi, les esquisses numériques qui sont manifestement moins «chargées» puisqu'elles sont plus grandes, contiennent les mêmes informations (identification des espaces, taille des murs, annotations, aménagements, etc.) que les croquis papier.

En outre, tous les participants arrivent au même stade de définition du bâtiment à l'issue de l'activité et tous les résultats sont jugés équivalents en termes de qualité et de quantité d'information. Il n'est dès lors pas possible d'identifier un réel effet de l'environnement numérique sur la qualité du travail.

Dans les activités sur papier virtuel avec interprétation, une différenciation entre calques contenant des dessins « brouillons » et « nets » est apparue. Dans les activités papier-crayon et sur le logiciel sans interprétation, chacun des calques contient conjointement des dessins de brouillon et des dessins au net. Ceci nous a amené à confirmer la présence de deux types d'esquisses utilisées dans la phase conceptuelle de la conception (que nous avions déjà identifiées dans [Safin et al. 2007] :

- L'esquisse de simulation. Il s'agit de dessins « de brouillon » caractérisés par un haut degré d'ambiguïté et de personnalisation et par une multiplication des solutions graphiques au problème architectural, qui rendent ardue voire impossible leur communication à autrui. Dans EsQUIsE, nous avons constaté que les architectes réalisent ces esquisses de simulation exclusivement en couleur et sur des calques dédiés afin d'éviter leur reconnaissance par le logiciel qui se traduirait par des incohérences dans l'interprétation.

- L'esquisse synthétique. Elle est issue d'activités régulières de mises au net. Le dessin est moins ambigu et plus conventionnel que les précédentes. Chaque frontière (mur, paroi vitrée...) est représentée par un trait unique et ces frontières servent à circonscrire des espaces différenciés et donc à arrêter un agencement spatial particulier. L'esquisse synthétique est en grande partie interprétable par un autre architecte. Dans EsQUIsE, ces esquisses n'apparaissent que sur des calques au net et sont dessinées en noir.

Au point de vue de la succession des dessins, on peut décrire les processus de la façon suivante

- Dans les activités sur outils traditionnels, on observe des itérations entre esquisses de simulation et d'esquisses synthétiques: les concepteurs dessinent des croquis de 
brouillon, qu'ils mettent au net, avant de les réutiliser eux-mêmes comme brouillons, qui seront à leur tour mis au net, etc.

- Dans les activités de croquis numérique sans interprétation, on voit évoluer globalement les représentations d'esquisses de simulation vers des esquisses synthétiques. Ainsi, les dessins de brouillon sont, au fur et à mesure du déroulement de l'activité, remplacés par des dessins nets.

- Dans les activités de croquis numérique avec interprétation, les deux types de dessins coexistent: les esquisses de simulation sont présentes sur certains calques et les esquisses synthétiques sur d'autres. Les concepteurs travaillent tour à tour sur une seule de ces catégories d'esquisses.

\section{RESULTATS : UTILISATION DES CALQUES ET SUPPORTS}

Les activités observées ont donné lieu à la définition de lignes du temps de l'utilisation des différents supports (calques, papiers...). Ces lignes du temps nous permettent d'appréhender le processus de conception dans son ensemble. Et d'en identifier des phases et des structures récurrentes. En ordonnée sont listés les différents supports (feuilles, calques et calques virtuels) et en abscisse le temps, arrondi à la minute.

Dans cette section nous montrons et expliquons les six lignes du temps correspondant à l'activité de conception des six participants.

\section{1. UTIILISATION DES CALQUES SUR PAPIER-CRAYON}

L'utilisation des calques lors des deux activités ayant pris place dans l'environnement traditionnel papier-crayon peut être représenté comme suit :

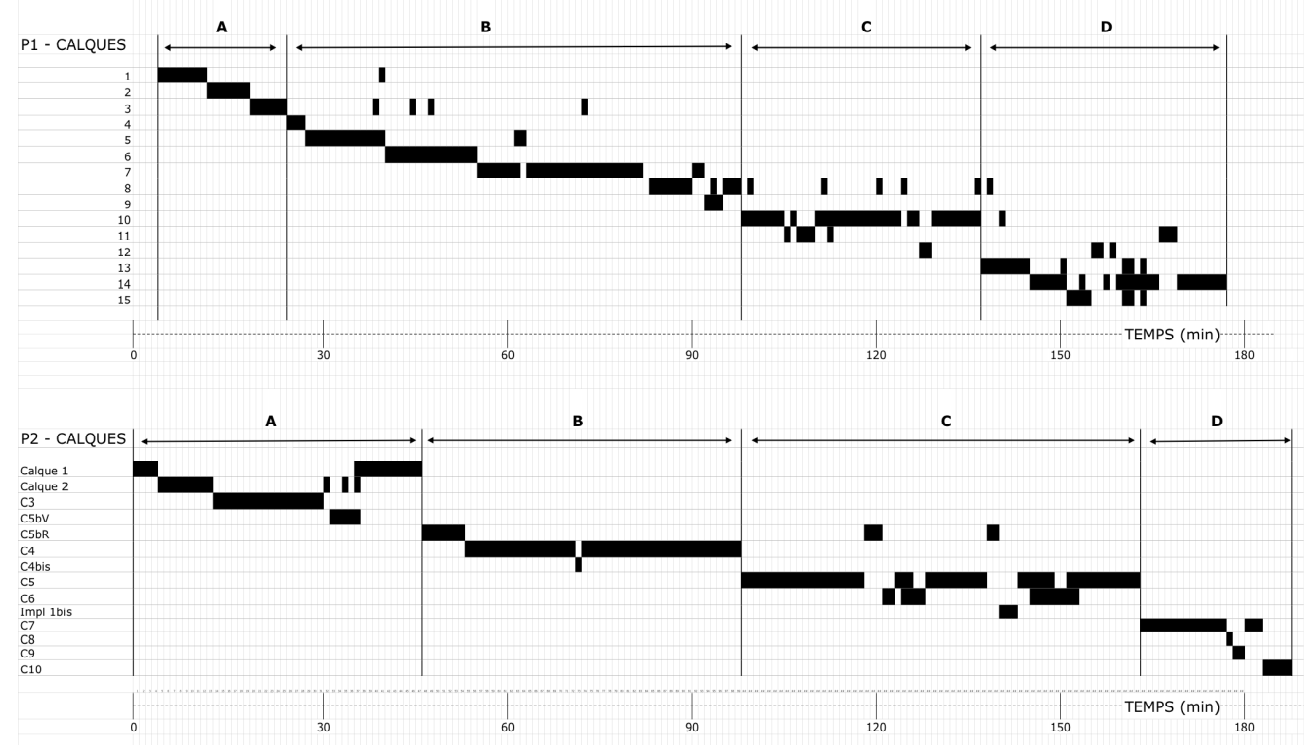

Figure 13 : ligne du temps de l'emploi des calques lors des activités des participants sur papier-crayon (P1 et P2). 
Ces activités sont relativement semblables : de nombreux calques sont utilisés et l'approche est « en escalier », c'est-à-dire que les calques sont rarement réutilisés. On peut identifier plusieurs phases, qui sont semblables pour les deux participants :

- Une phase d'exploration conceptuelle (phase A pour P1 et P2) consistant à poser les premières bases, à restructurer les données de l'énoncé, à imaginer une première forme du bâtiment dans son ensemble et à définir l'emprise au sol du bâtiment.

- Une phase de travail sur le rez-de-chaussée (phase B), consistant à résoudre l'essentiel des problèmes relatifs au rez-de-chaussée, d'en aménager précisément l'intérieur.

- Une phase de travail sur les étages (phase C), consistant à définir, en fonction des contraintes liées au rez-de-chaussée, les éléments architecturaux des étages. Cette phase comprend quelques retours en arrière sur le rez-de-chaussée afin de coordonner les différentes contraintes.

- Une résolution des problèmes finaux. Il s'agit, sur base des éléments précédents, de finaliser le bâtiment: aménagement intérieur et extérieur, éventuellement dessins de coupes ou perspectives.

Entre ces phases les calques sont peu ou pas réutilisés. Dans les premières phases, les concepteurs se concentrent sur un unique calque et un unique dessin, tandis que dans les deux dernières phases, ils travaillent sur plusieurs calques en parallèle. En effet, en fin de processus, il est nécessaire d'évaluer correctement l'impact de décisions relatives à une partie du bâtiment sur les autres parties de ce même bâtiment.

Les changements de calques correspondent au démarrage d'un nouveau dessin. On peut identifier plusieurs types d'activités lors d'un changement de calque :

- un nouveau dessin est créé à partir de rien ;

- un dessin précédent est recopié dans son intégralité avant de servir de base à un autre ;

- un dessin est partiellement recopié pour servir de base à un autre ;

Les deux derniers types peuvent être qualifiés de mise au net de dessin : le concepteur transforme ses dessins de brouillon en dessins au net. Une fois un dessin mis au net, il n'est plus modifié, même s'il est parfois consulté. Cette mise au net peut s'effectuer en se basant sur la transparence des calques ou en repartant d'une feuille blanche.

\section{2. UTIILISATION DES CALQUES SUR SKETSHA}

L'utilisation des calques lors des deux activités ayant pris place dans l'environnement numérique sans interprétation (logiciel SketSha) peut être représenté comme suit (figure 14). Les deux activités sont aussi tout à fait comparables. On retrouve les mêmes phases que sur papier-crayon : exploration (phase A), conception du rez(phase B), conception des étages (phase C) et finitions (phase D). Ici aussi les calques sont peu réutilisés entre les phases. Par contre, il y a beaucoup moins de calques : 5 et 7 sur SketSha contre 14 et 15 sur papiercrayon. La transparence des calques virtuels est régulièrement adaptée pour afficher plusieurs dessins simultanément. Lors des changements de calque, on voit aussi les mécanismes de mise au net. Les calques, une fois mis de côté, ne sont plus modifiés. Cependant, si les calques ne sont pas modifiés régulièrement, ils sont par contre utilisés comme support au plan en cours de modification, voire comme complément au dessin (en réglant les calques comme entièrement transparents). Enfin, à quelques rares exceptions 
près, les calques sont modifiés individuellement, les concepteurs travaillant rarement en parallèle sur plusieurs calques.

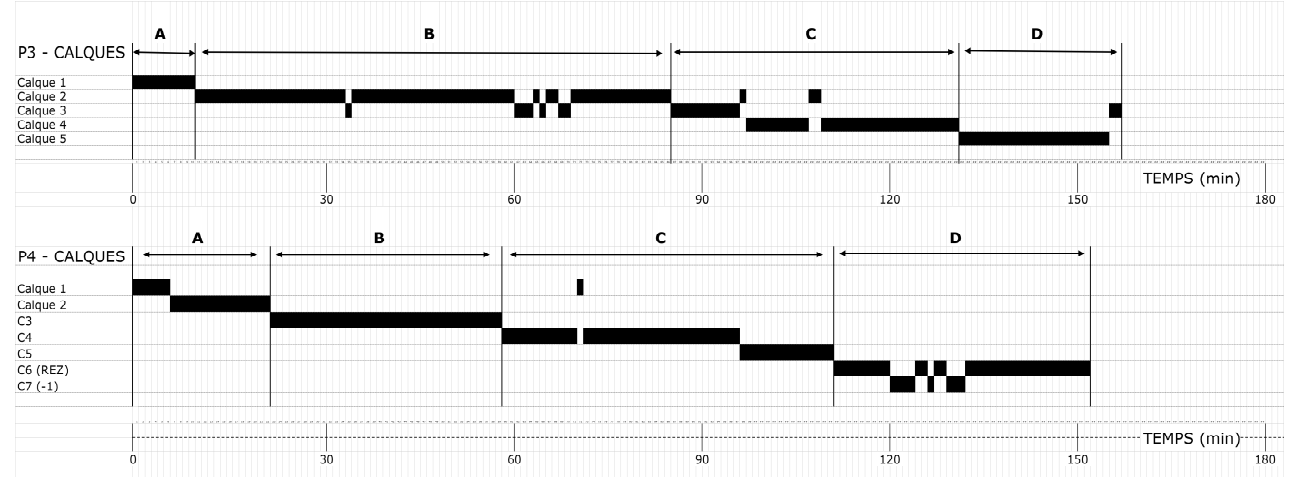

Figure 14 : Ligne du temps de l'emploi des calques lors des activités des participants sur SketSha (P3 et P4)

Comme évoqué précédemment, les dessins évoluent de représentations de brouillon vers des représentations synthétiques dans un mouvement global. Chaque esquisse précise le concept et est de plus en plus "propre». En outre, les activités sur SketSha sont globalement moins longues que sur les deux autres environnements.

\section{3. UTIILISATION DES CALQUES SUR ESQUISE}

L'utilisation des calques lors des deux activités ayant pris place dans l'environnement numérique avec interprétation (logiciel EsQUIsE) peut être représenté comme suit :

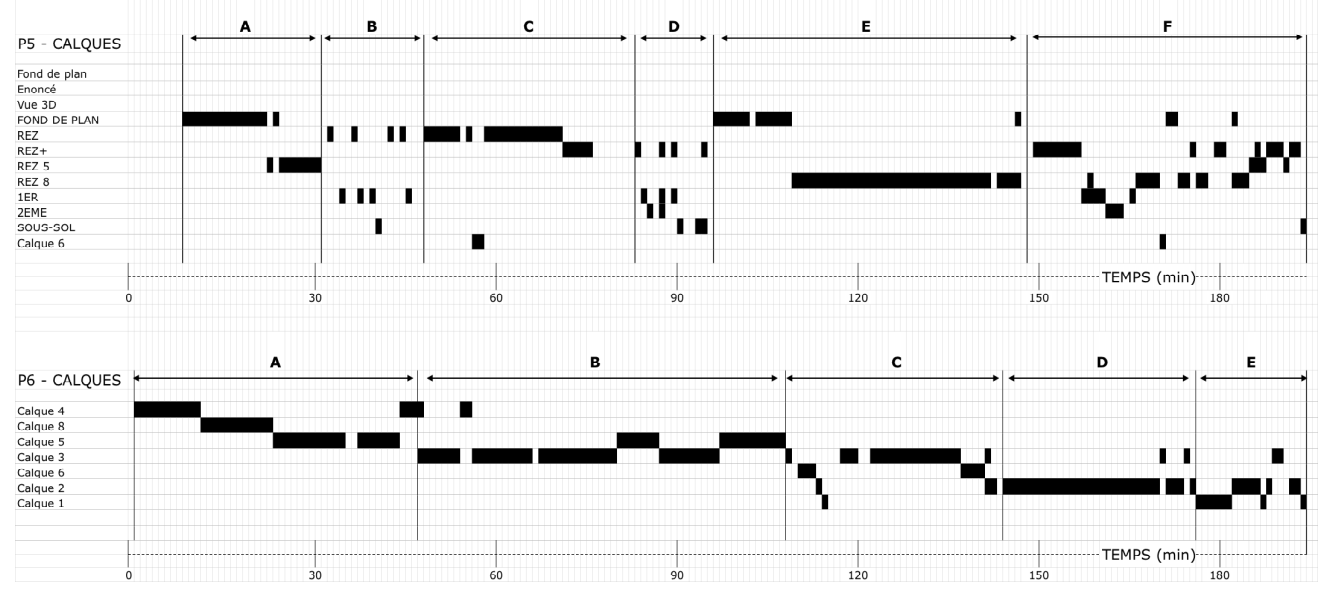

Figure 15 : Ligne du temps de l'emploi des calques lors des activités des participants sur EsQUIsE (P5 et P6) 
Sur EsQUIsE, la structure est manifestement différente de celle observée dans les deux autres environnements. Ici, outre la phase d'analyse présente aussi dans les deux activités (phases A), on voit apparaître deux types de phases très différenciées :

- des phases de travail mono-calque durant lesquelles le concepteur travaille principalement sur un calque (ou alterne de longues séances de travail sur deux calques) : phases $\mathrm{C}$ et $\mathrm{E}$ pour P5 et phases B et D pour P6 ;

- des phases de travail multi-calques durant lesquelles le concepteur travaille sur plusieurs calques en même temps, en passant d'un à l'autre très rapidement : phases $\mathrm{B}, \mathrm{D}$ et $\mathrm{F}$ pour P5 et phases C et E pour P6.

Lors des premières, le concepteur travaille exclusivement sur des calques de brouillon, tandis que dans les phases multi-calques, il modifie l'ensemble de ses calques «nets». Ainsi, l'activité de mise au net de dessins n'est plus ici une composante du dessin, mais bien une phase à part entière. La mise au net de tous les dessins est effectuée à des étapes-clés. C'est lors de ces étapes de mise au net, et uniquement lors de celles-ci, que le modèle 3D auto-généré est appelé, consulté et manipulé.

Cette structure s'explique par les modalités du dialogue homme-machine: pour bénéficier d'un modèle 3D propre et cohérent, le concepteur doit disposer de l'ensemble de son bâtiment interprété, c'est-à-dire que tous les étages doivent être dessinés au trait noir, et de manière peu ambiguë. Ainsi, il met au net tous ses calques au moment où il souhaite visualiser l'interprétation de son bâtiment par le logiciel.

Ici aussi on retrouve un nombre de calques proches de celui observé dans l'environnement numérique sans interprétation : 9 pour P5 et 7 pour P6.

\section{4. COMPARATIFS}

Les différentes lignes du temps et les descriptions du processus montrent clairement des différences entre les trois environnements. On peut synthétiser les processus de la façon suivante (figure 16)

Plusieurs éléments permettent de distinguer les trois processus.

- Le nombre de calques. On observe l'utilisation de moins de calques dans les environnements numériques. Par contre cette réduction du nombre de calques s'accompagne de la coexistence de plusieurs dessins sur un même support. Plusieurs facteurs participent à cette différence de comportements : la dimension des calques n'est pas limitée (on n'est donc jamais obligé de changer de support par manque de place) et la transparence des calques est à tout moment modulable, ce qui permet de voir dynamiquement même les traits les plus fins des supports inférieurs ou, au contraire, de travailler sur un support opaque.

- La non réutilisation des calques. Sur papier ou avec le logiciel SketSha, nous observons un graphe «en escalier» qui témoigne du fait que le concepteur ne revient pas sur les calques précédents. Sur EsQUIsE, on observe le même phénomène en ce qui concerne les calques de brouillon mais les calques nets, destinés à l'interprétation $3 \mathrm{D}$, sont réutilisés. Il existe de nombreuses représentations plus ou moins abstraites ou abouties du bâtiment mais le concepteur ne manipule qu'un seul modèle de son projet. 

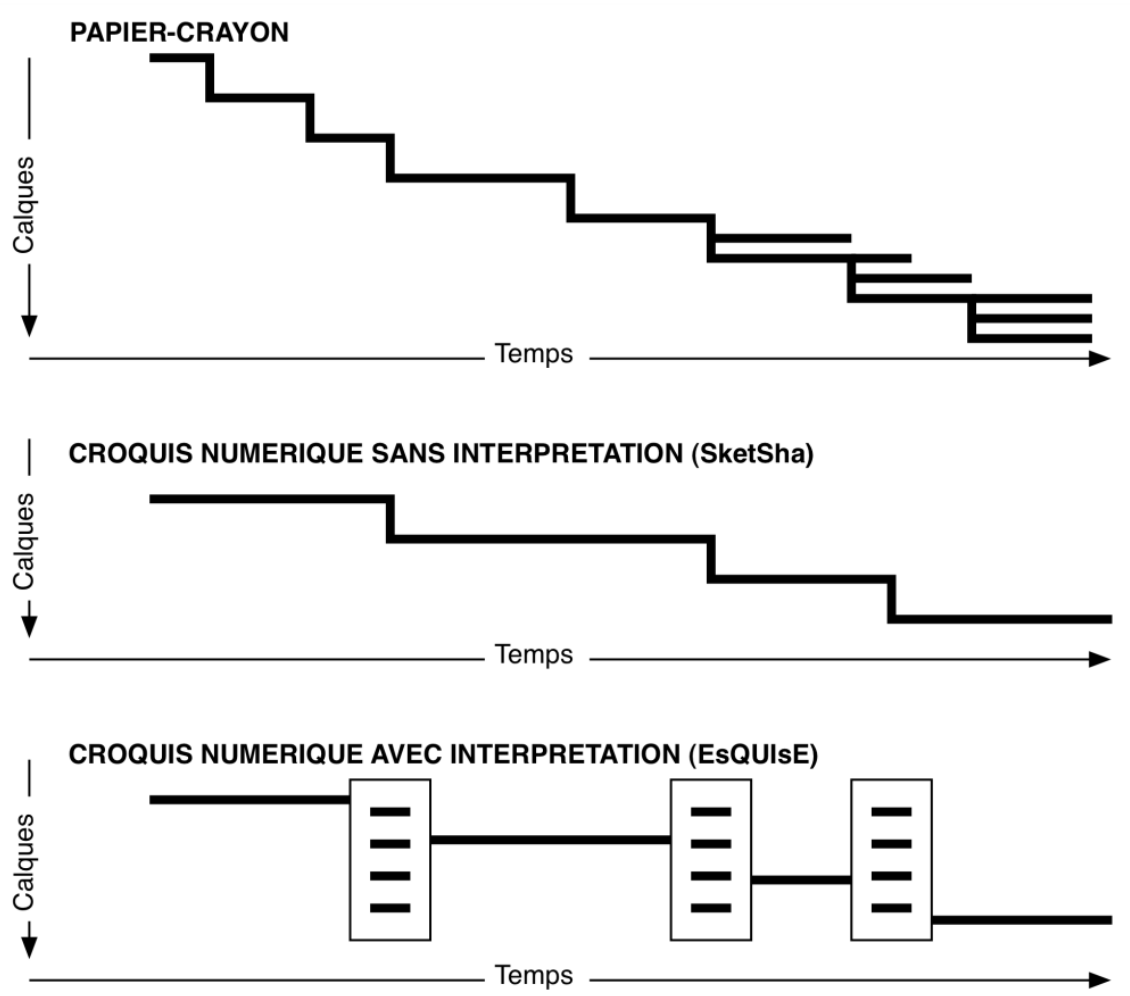

Figure 16 : Comparaison des processus schématiques dans les trois environnements.

- La différenciation des calques. Lors du travail sur papier, un croquis réalisé au net sert de base à la poursuite de la simulation et devient donc de ce fait un croquis de brouillon. Ce brouillon sera à son tour remis au net sur le même support ou lors d'un changement de calque et le processus recommencera. On observe donc une coexistence de croquis de brouillon et de croquis nets sur le même support. EsQUIsE, au contraire, favorise la différenciation de ces deux types de représentations : d'une part des calques brouillons et d'autre part des calques nets.

- Utilisation d'un calque à la fois. Malgré une réflexion approfondie sur la notion de calque numérique et sur l'ergonomie de leur manipulation, l'environnement numérique n'offre pas la même souplesse que les feuilles de papier. Par exemple, il n'est pas possible actuellement de disposer côte à côte deux documents. Ainsi, plutôt que de manipuler régulièrement les onglets des calques pour en changer, le concepteur préfère ne travailler que sur un seul calque à la fois pour concevoir, et utilise intensément la transparence.

- Structure du travail due à l'interprétation. Lors du dessin avec le logiciel EsQUIsE, on observe des phases très caractéristiques de travail sur plusieurs calques à la fois. Elles correspondent à la transcription des idées issues des esquisses de simulation pour l'interprétation par le système et la génération du modèle 3D.

\section{RESULTAT : PROCESSUS DE MISE AU NET}


Dans cette section, nous nous intéressons aux activités cognitives sous-tendant le processus de mise au net, considéré ici comme l'activité visant à transformer une esquisse de brouillon en dessin net. La dynamique de cette activité diffère suivant les trois environnements.

\section{1. MISE AU NET SUR PAPIER-CRAYON}

Dans les activités sur papier-crayon, nous observons différents mécanismes et fonctions de la remise au net.

1. Choisir un tracé. Sur le dessin de brouillon (figure 1), les éléments architecturaux sont fréquemment délimités par plusieurs traits, plus ou moins superposés, plutôt que par des contours simples. Lors de la mise au net, ces traits sont remplacés par un tracé unique, généralement plus épais (voir figure 2). De cette opération découle donc une simplification du dessin et une réduction du flou graphique. Cela consiste à remplacer un groupe de traits par un seul. Mais nous observons qu'il ne s'agit pas uniquement de retracer la solution la plus saillante. Il s'agit surtout de poser un choix conscient : sélectionner une seule position pour un objet quand le dessin de brouillon en présentait plusieurs. Ce choix n'engage cependant pas le concepteur pour l'entièreté du processus : le dessin remis au net pourra de nouveau devenir brouillon et ces choix pourront être remis en cause. Cependant la mise au net aura permis de figer certains éléments, de donner un contexte provisoire à l'activité de conception.

2. Dessiner à la règle. Le dessinateur abandonne le dessin à main levée préférant utiliser une règle pour tracer des segments de droite. Il s'agit donc ici aussi d'un mécanisme de réduction du flou et de l'imprécision du tracé. Le trait se rapproche de la projection de l'objet architectural qu'il figure (par exemple le mur).

3. Préciser les mesures. Le concepteur affecte une mesure précise aux traits lors de la remise au net. Il ne s'agit pourtant pas d'une action de conception mais d'une mesure de vérification. Le dessin de brouillon découlait déjà d'une réflexion sur les dimensions mais le flou et l'imprécision de cette représentation ne permettaient pas de contrôler les contraintes géométriques liées au programme architectural. La réduction du flou graphique s'accompagne d'une réduction de l'imprécision et souvent d'une vérification fonctionnelle des dimensions.

4. Expliciter le fonctionnement. La mise au net s'accompagne aussi d'ajout de légendes permettant de situer les locaux. De plus, on observe l'ajout d'informations : représentation du mobilier, position des portes, annotations etc. La vitesse d'exécution de cette tâche, quasi automatique, nous laisse croire qu'il s'agit d'une opération d'explicitation (réduction de l'ambiguïté) plutôt qu'une opération de conception. L'aménagement intérieur n'est pas conçu à ce stade, le dessin du mobilier permet simplement de donner des indications sur le fonctionnement du bâtiment.

5. Utiliser la gomme. Les concepteurs utilisent parfois la gomme sur le dessin au net, ce qui n'est pas le cas pour les dessins de brouillon. Pour ces derniers, ils laissent coexister des traits de toutes sortes et des solutions multiples. La correction se fait par incrémentation du croquis.

6. Surligner. Un des deux participants surligne à plusieurs reprises à l'aide d'un marqueur épais les traits de certains locaux. Un local dont le contour est surligné est temporairement "gelé »: le concepteur n'y travaillera plus avant un certain temps. Ce surlignage n'est pas précis (trait épais et tracé à main levée) mais concerne toujours des éléments issus d'une mise au net précédente. 
Ces six éléments montrent que la remise au net fait évoluer le dessin d'une représentation floue et imprécise, que nous pouvons qualifier de conceptuelle, vers une représentation précisée géométriquement. Cette réduction de l'incertitude du dessin est une des caractéristiques fondamentales du processus de conception [Lebahar, 1983].

On observe en outre deux types de mise au net. Le premier, évoqué ci-dessus, consiste à opérer un choix parmi plusieurs solutions. Ce choix, éventuellement validé par une vérification dimensionnelle, est retranscrit dans une plus grande précision géométrique. Le second type de mise au net consiste uniquement à retranscrire le dessin « au propre ». Ici les traits sont repassés finement, sans augmentation de la précision et sans vérification dimensionnelle. Il s'agit simplement de reporter des éléments non aboutis d'une représentation à une autre. Les éléments sont simplement retranscrits, ils feront l'objet de réflexions de conception plus tard dans le processus. Lorsque le concepteur utilise un nouveau calque pour redessiner le projet, ce calque peut être à la fois composé d'éléments mis au net (sélection consciente parmi les possibles) et d'éléments simplement retranscrits (dupliqués). Cette méthode lui permet de valider certaines parties du projet sans attendre la cohérence complète de la représentation.

Enfin, nous observons chez les deux participants que l'activité de mise au net n'est pas clairement dissociée de l'activité de conception: les moments de mise au net sont entrecoupés de moments de conception.

\section{2. MISE AU NET SUR SKETSHA}

Dans l'environnement numérique sans interprétation, le processus de mise au net à proprement parler ne prend part que dans la première moitié de l'activité environ. Les dessins sont modifiés et dupliqués de la même façon qu'avec le papier-crayon : le nombre de traits se réduit, les traits se précisent, des choix sont opérés, des mesures sont prises. Néanmoins, dans la seconde moitié de l'activité, les dessins sont uniquement nets. Ainsi, les changements de calques s'accompagnent d'une duplication d'éléments nets : l'emprise du rez-de-chaussée pour la conception de l'étage, par exemple. La recherche conceptuelle semble donc plus mentalisée : il y a moins d'expression graphique directe dans la seconde moitié de l'activité, et les décisions sont prises par le concepteur avant de les retranscrire sur papier. On peut considérer l'expression graphique comme une mise au net directe de la recherche mentale, dans un souci d'économie du dessin.

\section{3. MISE AU NET SUR ESQUISE}

Comme évoqué précédemment, nous observons une méthode de travail très particulière liée aux activités de conception dans notre environnement numérique avec interprétation. Alors que l'essentiel de l'activité se déroule sur un seul calque, nous identifions des séquences ponctuelles lors desquelles l'ensemble des informations d'un calque «brouillon » est mis au net sur des calques «nets». Autrement dit, de longues séquences de simulation sur un calque unique sont ponctuées par des moments de remise au net lors desquelles tous les étages du bâtiment sont redessinés en même temps, en vue d'une interprétation par le logiciel. L'activité de mise au net est bien circonscrite dans le temps et peut donc être comprise comme une " phase» du travail de conception. Lors de ces phases, le concepteur remet toujours au net l'entièreté des plans. En effet, contrairement à la mise au net sur papier qui peut ne concerner qu'une partie du plan ou être interrompue par des actions de 
conception, l'activité des concepteurs sur EsQUIsE consiste à produire, en une fois, une représentation complète et cohérente du bâtiment. Nous décrivons ci-dessous plus en détails les opérations menées lors de ces "phases de mise au net». Un exemple de calque numérique brouillon et de calque numérique net sont donnés aux figures 3 et 4 .

Comme s'il travaillait sur papier, le concepteur effectue la remise au net de certains traits à la règle et vérifie précisément ses mesures. Par contre, entre les éléments remis au net et les brouillons, on constate une moins bonne correspondance que sur papier. Certains éléments retranscrits ne sont pas directement issus du brouillon. Cela s'explique notamment par le fait que le dessin de tous les étages nécessite, en vue de faciliter l'interprétation logicielle, des opérations de coordination entre les niveaux qui sont reportées à plus tard dans l'activité sur papier. Le concepteur gère donc son espace de dessin en partie comme sur papier : un dessin, remis au net devient un espace de simulation. Par contre il organise son temps de manière différente en réalisant la mise au net exhaustive de tous les étages en une seule étape.

Après une phase de simulation, les concepteurs ne recréent pas de nouveaux dessins au net. Ils utilisent la possibilité offerte par le système d'effacer des traits et corrigent les plans au net déjà tracés, conformément au nouvel état d'avancement du projet. Ils établissent ainsi un lien direct entre les représentations nettes et l'interprétation qu'en opère le logiciel (ici une maquette 3D auto-générée). Ceci est particulièrement frappant pour le participant $\mathrm{P} 5$, qui ne remet au net que les contours des locaux. Il ne redessine pas le mobilier et n'ajoute pas de légendes. Il s'agit donc bien de reproduire uniquement ce qui est interprété par le système. P6 ajoute quelques légendes en couleurs, non interprétées par le logiciel.

Dans le cas des participants travaillant sur EsQUIsE, il apparaît clairement que l'activité d'interprétation du logiciel (la génération de la maquette 3D) influence fortement la méthode de travail : la remise au net dans EsQUIsE n'a pas uniquement un rôle « interne » pour le concepteur, mais est un élément central du dialogue homme-machine. Elle est utilisée pour décrire le bâtiment à la machine en vue de son interprétation, notamment pour la génération du modèle 3D. C'est sans doute pour cette raison que les concepteurs sont attentifs à retracer des plans complets (plutôt que la partie qui les intéresse à un moment donné) et tous les niveaux dans la même étape. Cela permet de vérifier l'interprétation du logiciel et la cohérence du modèle de bâtiment généré.

\section{4. COMPARAISON DES ACTIVITES DANS LES TROIS ENVIRONNEMENTS}

On retrouve des différences et des similitudes dans l'ensemble de ces activités. Pour synthétiser, on note que les opérations de transformations du dessin de brouillon en dessin net sont les mêmes dans les six activités :

- une simplification graphique opère par réduction du nombre de traits, soit en choisissant un trait parmi plusieurs possibles, soit en ne recopiant pas dans la mise au net certains éléments graphiques (hachures, annotations etc.) Cette opération permet de réduire le flou de l'esquisse, mais ne s'accompagne pas nécessairement d'une réduction de l'imprécision.

- une réduction de l'imprécision s'observe de manière très claire lors des mises au net à la règle qui s'accompagnent très souvent de vérifications dimensionnelles (largeur d'un couloir, surface d'un local, recul du bâtiment, etc.). 
- une réduction de l'ambiguïté s'opère par une évolution du dessin vers des conventions graphiques partagées ou par l'explicitation du contenu par ajout de légendes ou de symboles.

- une sélection d'une solution. Sur les esquisses de brouillon, il n'est pas rare de voir coexister plusieurs solutions architecturales. La mise au net opère un choix parmi différents possibles présents dans l'esquisse de simulation.

Par contre, les objectifs de ces transformations ne sont pas les mêmes dans les différents environnements. Dans tous les cas il s'agit d'arrêter temporairement une version du bâtiment. Lors du travail sur papier et sur SketSha, la mise au net vise avant tout à réduire la complexité du problème architectural, en passant de dessins aux solutions multiples et imprécises à un dessin présentant une solution unique. Sur EsQUIsE, cette mise au net vise à dialoguer avec la machine, à décrire le plan en vue de l'interprétation et, en retour, en recevoir des évaluations (le modèle 3D). C'est donc principalement à cette étape, outre la sélection d'une solution, que le concepteur procède à la vérification de la solution.

Par ailleurs, la gestion de l'espace virtuel de dessin composé de l'ensemble des calques est différente dans l'activité sur papier et sur ordinateur. Sur papier, les calques se succèdent et reçoivent des représentations nettes qui deviennent ensuite une base pour l'activité de simulation. On assiste donc à la coexistence des deux types de représentations sur la même feuille. Au contraire, les calques numériques sont spontanément dédiés à l'une ou l'autre de ces activités.

La gestion du temps est aussi différente selon les supports utilisés. Les périodes de simulation et de remise au net alternent sur papier. Le concepteur passe aisément de l'une à l'autre, changeant ou non de calque. Par contre, sur EsQUIsE, le concepteur engagé dans une phase de remise au net poursuit cette activité jusqu'à l'obtention de tous les plans du bâtiment.

\begin{tabular}{|c|c|c|c|}
\hline & Papier-crayon & SketSha & EsQUIsE \\
\hline Objectif & $\begin{array}{l}\text { Réduire la complexité } \\
\text { du croquis }\end{array}$ & $\begin{array}{l}\text { Réduire la complexité } \\
\text { du croquis }\end{array}$ & $\begin{array}{l}\text { Décrire le projet en vue } \\
\text { de la génération du } \\
\text { modèle 3D }\end{array}$ \\
\hline $\begin{array}{l}\text { Gestion de } \\
\text { l'espace }\end{array}$ & $\begin{array}{l}\text { Coexistence sur un } \\
\text { même calque de dessins } \\
\text { nets et brouillons }\end{array}$ & \begin{tabular}{llr}
\multicolumn{2}{l}{ Spécialisation } & des \\
calques (nets & ou \\
brouillons) & & \\
\end{tabular} & \begin{tabular}{llr}
\multicolumn{2}{l}{$\begin{array}{l}\text { Spécialisation } \\
\text { calques (nets }\end{array}$} & des \\
brouillons) & & \\
\end{tabular} \\
\hline $\begin{array}{l}\text { Gestion } \\
\text { du temps }\end{array}$ & $\begin{array}{l}\text { Alternance } \\
\text { simulations et remises } \\
\text { au net sur un même } \\
\text { calque }\end{array}$ & $\begin{array}{l}\text { Uniquement des dessins } \\
\text { nets dans la seconde } \\
\text { partie de l'activité }\end{array}$ & $\begin{array}{l}\text { Remise au net de tout le } \\
\text { bâtiment en une seule } \\
\text { fois }\end{array}$ \\
\hline
\end{tabular}

Tableau 3 : récapitulatif des différences dans la mise au net de dessins.

\section{DISCUSSION}

L'étude de l'activité de remise au net nous a permis de mieux appréhender l'activité de conception préliminaire par dessin d'esquisse en architecture. Les différences observées entre les conceptions réalisées sur papier et avec les logiciels que nous développons nous 
permettent aujourd'hui de dégager quelques pistes pour le développement de systèmes d'IHM à base d'esquisse, avec ou sans interprétations.

\section{1. INTERETS ET LIMITES DES INTERFACES-ESQUISSES POUR LA CONCEPTION}

De nombreux auteurs ont mis en avant l'intérêt de l'esquisse en conception préliminaire, par opposition aux outils de CAO. La souplesse de l'interaction [Bilda et Damakian 2002], l'expressivité du croquis [Rogers et al. 2000; MacCall et al. 2001] sont des atouts indéniables de ce mode d'expression. D'autres auteurs ont montré l'intérêt des interfaces tangibles, dont le stylo est une forme rudimentaire, comme vecteur d'un plus grand investissement corporel dans la recherche de solutions, ce qui est reconnu comme facilitant la cognition spatiale [Kim \& Maher, 2008]. Néanmoins, peu d'études se sont penchées sur l'influence de la numérisation du croquis, c'est-à-dire la comparaison entre le croquis papier et le croquis numérique.

Tout d'abord, il nous faut noter que l'utilisation d'interfaces-esquisses est une voie prometteuse pour soutenir la conception. En effet, notre étude montre que la numérisation du croquis ne change pas fondamentalement ses caractéristiques intrinsèques, ni son utilité : on retrouve globalement les mêmes caractéristiques aux dessins et les mêmes procédures et comportements de mise au net. La transposition dans un environnement numérique offre en plus l'avantage de l'augmentation du croquis. Ainsi, outre les fonctions d'édition et de manipulation (le zoom et la translation permettent de disposer d'une feuille de taille virtuellement infinie), les logiciels que nous développons proposent deux types d'augmentations : des évaluations précoces de performance sur base d'une interprétation dans le cas d'EsQUIsE et un partage du dessin à distance et en temps réel dans le cas de SketSha. Garder des modes d'expression naturels tout en profitant de la puissance de calcul de l'informatique est un défi qui semble réalisable dans le domaine de la conception.

Cependant, l'approche numérique présente certaines limitations car les potentialités d'expressivité graphique sont réduites dans nos environnements logiciels. Nous avons montré que le dessin numérique, s'il contenait quantité d'informations comparable au dessin papier, était composé de moins de traits, avec moins de variations, et que spontanément, les concepteurs étaient moins expressifs dans leurs productions graphiques. Si cette différence dans le type de dessins est aisément compréhensible dans le cas d'un logiciel d'interprétation de croquis (car il est nécessaire d'être explicite et précis pour se faire comprendre de la machine), cette observation est plus surprenante dans le cas de SketSha où le concepteur est libre de dessiner de la façon la plus souple pour lui. Or, le dessin de brouillon a tendance au fil de l'activité à disparaître au profit de dessins exclusivement nets. Nous proposons deux hypothèses pour expliquer ce constat :

- La première est liée l'effort nécessaire pour produire le dessin. Le dessin dans l'environnement numérique est manifestement coûteux : la taille du dessin nécessite d'amples mouvements de la part du concepteur, les types de traits limités restreignent aussi ses potentialités expressives et la vitesse du dessin est réduite par le stylo numérique (qui est un peu moins maniable que le stylo habituel). Ce coût implique pour le concepteur de «rentabiliser» son dessin: il n'est pas envisageable de créer une représentation pour la «jeter» l'instant d'après. Tout dessin doit donc posséder un caractère relativement définitif. 
- La seconde hypothèse que nous faisons est liée à la transparence. Comme évoqué précédemment, les concepteurs sur SketSha utilisent beaucoup la transparence des calques. Or, pour pouvoir tirer profit de cette fonctionnalité, il est nécessaire que les dessins ne soient pas trop surchargés. Le concepteur limiterait donc le nombre de traits de ses dessins pour permettre un travail en transparence. En outre, le logiciel offre la possibilité de rendre les calques totalement transparents. Ainsi, il est possible non plus d'uniquement superposer plusieurs dessins, mais aussi véritablement de les fusionner. Il n'est donc plus nécessaire pour le concepteur que l'information soit redondante d'un calque à l'autre, car il peut utiliser deux dessins en même temps.

Ces résultats sont cohérents avec ceux de [Koutamanis 2005] qui, en étudiant l'utilisation du stylo numérique Anoto dans le cadre de croquis d'architecture, constate que les possibilités d'expression graphique plus réduites rendent l'esquisse numérique mieux adaptée aux dessins abstraits comme les plans ou les diagrammes qu'aux esquisses plus artistiques.

En conclusion, nous suggérons que les IHM à base de stylo pour la conception, basés sur une analogie avec les outils traditionnels, doivent soutenir les qualités essentielles que sont la fluidité, la souplesse d'utilisation et l'expressivité graphique. L'observation de l'activité sur papier montre que l'outil doit proposer différents types de traits (crayons, feutres etc,...), de différentes couleurs, sans forcer de sémantique attachée à chaque type de trait. Le logiciel doit permettre un mode de fonctionnement de type palette graphique. L'utilisation d'une règle - réelle ou virtuelle - doit être rendue possible pour permettre les opérations la réduction de l'imprécision. Il nous semble aussi primordial de soutenir la coexistence des deux types de dessins identifiés. Néanmoins, nos observations sur SketSha montrent que ce fonctionnement de type palette graphique n'est pas suffisant. Pour réellement adopter la souplesse du dessin, il est nécessaire d'une part d'offrir au concepteur des traits d'une grande variété, lui permettant d'effectuer son « coup de crayon », mais aussi de lui permettre de manipuler simultanément des documents en même temps. Ainsi, il nous semble nécessaire de faire évoluer l'interaction du papier virtuel à l'espace de dessin virtuel. Des réflexions sur les modalités de manipulation de documents multiples au stylo et sur la création de zones de travail différenciées (notamment dans les applications collaboratives) doivent être menées, accompagnées éventuellement de réflexions sur l'usage d'interactions gestuelles en complément du stylo.

\section{2. DIFFERENTS TYPES D'ESQUISSES ET MODES DE TRANSFORMATIONS}

Notre étude a montré que la phase dite d'esquisse est caractérisée par des productions graphiques variées. Il n'existe pas qu'une seule esquisse en conception préliminaire, mais bien deux: l'esquisse de brouillon (ou conceptuelle) et le dessin net (ou esquisse synthétique).

L'esquisse de brouillon permet de concevoir sans choisir. L'architecte, grâce à l'imprécision et au flou de l'esquisse, ne fige pas son projet mais au contraire autorise la coexistence sur un même dessin de plusieurs solutions parfois contradictoires. À l'heure actuelle, ce genre de mécanisme n'est pas possible dans les outils de CAO classiques qui contraignent l'utilisateur à manipuler une solution unique qui va évoluer jusqu'à la fin de la conception [Juchmes et al. 2004]. L'esquisse de brouillon est personnelle et difficilement compréhensible par un agent extérieur, et donc à fortiori par un système informatique. 
La remise au net permet de choisir (sans nécessairement concevoir). Elle permet de passer d'une représentation où de multiples solutions coexistent, à la représentation d'un objet architectural unique. Il s'agit donc de choisir la meilleure option dans l'ici et maintenant de la conception. Cependant, l'esquisse synthétique n'est pas une représentation figée de l'objet architectural : le dessin mis au net est un instantané à un moment de la conception. Son objectif est de permettre la communication de manière un peu plus univoque des principales caractéristiques du projet en cours, grâce à des mécanismes simples de réduction de l'ambiguïté et de l'incertitude et des procédures de vérification, tout en étant ouvert au prolongement des idées, à leur critique et leur modification, donc à la continuation de la conception. Cette communication est à destination d'un collaborateur ou du concepteur lui-même. Ce type de dessins convient à l'interprétation par un logiciel.

La signification des traits imprécis du dessin de brouillon n'est pas modifiée lors du changement de représentation. La mise au net ne consiste cependant pas uniquement en une duplication de certains traits, elle peut s'accompagner d'une explicitation du projet (réduction de l'ambiguïté) par exemple en ajoutant des légendes au dessin. Par contre une modification de la topologie des espaces, de la forme, l'ajout ou le déplacement d'un élément architectural est une opération de conception. Cela ne signifie cependant pas que la mise au net est indépendante de la conception. Choisir de redessiner ou de surligner, choisir une échelle de dessin, changer de couleur de trait, sont des actes qui participent activement au processus mais pas par une action directe sur l'objet projeté. Cependant, dans le cas du travail en croquis numérique interprété, une solution complète et cohérente doit exister pour permettre la génération de la maquette $3 \mathrm{D}$. Cette contrainte supplémentaire implique, en plus des opérations de mise au net, de convoquer les processus cognitifs de vérification de la cohérence de l'objet, qui relèvent directement des activités de conception.

Ainsi, il nous apparaît que dans le cadre de l'interprétation de dessins, le système ne doit pas tenter de tout interpréter en temps réel. La différenciation entre les dessins nets et de brouillon, et la correspondance de ceux-ci entre le papier-crayon et l'esquisse numérique nous invitent à penser que le paradigme de l'interprétation complète de l'esquisse de conception est quelque peu illusoire. Au-delà de la difficulté technique (l'esquisse de simulation est par nature incomplète et incohérente), nos observations montrent que le dessinateur a tendance à se protéger du regard du système en utilisant des couleurs non interprétées par le logiciel jusqu'au moment où il décide d'expliciter son travail au système. L'esquisse de brouillon est, et doit rester, un lieu privilégié d'exploration personnelle de solutions, sans aucune contrainte. En revanche l'esquisse nette, instrument de communication, semble particulièrement appropriée à une interprétation numérique.

\section{3. INFLUENCES DE L'INTERPRETATION DU DESSIN ET DE LA 3D SUR L'ACTIVITE}

Il est indéniable de constater que l'interprétation du croquis numérique, ainsi que la création en temps réel d'un modèle $3 \mathrm{D}$, influencent fortement l'activité graphique, et en particulier la mise au net. La présence d'une interprétation induit chez le concepteur un focus très fort sur le modèle 3D, structurant en partie son activité. Ce constat a déjà été réalisé par [Lim2003] qui, sur base d'une analyse des temps de travail associés aux différentes représentations utilisées par le concepteur sur papier-crayon et sur croquis numérique augmenté par la visualisation $3 \mathrm{D}$, constate que les proportions de temps de travail sur différents types de représentations (plans, coupes etc.) est modifiée. En particulier, la modélisation 3D générée 
mobilise une proportion conséquente de l'activité. Il ne s'intéresse cependant pas aux mécanismes de transformation des croquis. Pour bénéficier d'une maquette virtuelle 3D utile, il est nécessaire de proposer un modèle unique du bâtiment, qui soit complet, cohérent et suffisamment précis pour être compris par le logiciel. On retrouve ici toutes les caractéristiques du dessin «net» présent sur papier-crayon. Mais ces contraintes impliquent que ce modèle soit complété et précisé à des moments spécifiques, durant les "phases » observées de mise au net. En outre, ces phases constituent plus que le recopiage et les choix observés dans la mise au net: il s'agit aussi d'étapes de validations et d'évaluations explicites, notamment sur base de la 3D. Par ailleurs, EsQUIsE modifie aussi en partie le lieu de simulation : alors que les dessins nets et de brouillon sont entremêlés sur les plans papier, ils sont séparés, ou à tout aumoins en partie, sur les calques électroniques d'EsQUIsE. Ceci provient aussi sans doute du fait qu'il est important de produire un modèle « épuré » pour faciliter l'interprétation logicielle.

Ainsi, l'interprétation logicielle d'EsQUIsE n'imposerait pas de contraintes sur le contenu, mais bien sur la structure, c'est-à-dire le temps et l'espace dans lesquels s'inscrit la conception :

- les moments de mise au net qui, au lieu de s'inscrire dans le cours de la conception, s'en détachent pour devenir des phases à part entière ;

- les lieux de mise au net, l'outil logiciel invitant à dessiner les esquisses de brouillon et nets sur des calques différents.

Pour éviter les ruptures de l'activité créative qu'impose la nécessité d'être compris par le logiciel, il nous apparaît nécessaire de tenir compte de la dynamique de l'activité de conception. Ainsi, au lieu de "forcer" l'utilisateur à modifier les rapports spatiaux et temporels qu'il entretien avec ses dessins, il nous semble plus utile de se reposer sur la dynamique naturelle du concepteur en activité. On pourrait ainsi imaginer que l'interprétation ne prenne place qu'à des moments de mise au net. Ainsi, les mouvements récurrents de conception-mise au net observables sur papier-crayon devraient être respectés. Les moments de conception doivent rester le propre du concepteur, sans intervention ou interruption du logiciel, alors que les moments de mise au net, qui ne font pas intervenir un flux de pensée créatif à proprement parler, mais sont caractérisés par une série de choix conscients, pourraient être le bon moment pour une interprétation.

En outre, dans le cadre de la génération d'un modèle $3 \mathrm{D}$, nous devons nous garder de transformer un outil de support à la conception en modeleur explicite, même si les opérations sont réalisées au stylo. Nous avons observé que la possibilité de disposer de vues $3 \mathrm{D}$ modifiait l'activité du dessinateur en le poussant à remettre au net l'entièreté du dessin plutôt que de travailler de manière locale, en fonction de ses besoins. Cette observation nous pousse à croire que la génération du modèle 3D (et les retours d'interprétation de manière générale) ne doit être réalisée que sur demande et que ces informations complémentaires ne doivent pas être affichées à l'écran en permanence. Des études complémentaires sur l'usage de la 3D d'EsQUIsE [Mayeur, Leclercq \& Darses 2007] ont montré que le modèle 3D n'est pas très utilisé par les architectes professionnels, capables de s'imaginer la volumétrie d'un bâtiment sans difficulté.

\section{PERSPECTIVES: IMPLICATION POUR LE DEVELOPPEMENT DE LOGICIELS}


Il convient tout d'abord de souligner les limitations de l'étude. Le nombre réduit d'observations et le public observé (étudiants) ne nous permettent évidemment pas de tirer des conclusions définitives. Cependant, nos observations nous apparaissent suffisantes pour proposer des perspectives en vue de développements futurs ainsi que des propositions d'études complémentaires.

Dans le cadre du développement de nos deux logiciels, il est important de proposer une large palette d'outils de dessins (crayons de couleurs, feutres, surligneurs épais, etc...) afin de se rapprocher de la liberté d'expression graphique rencontrée sur papier.

Dans le cadre de SketSha, en plus d'un module de tracé réaliste, nous développons différents modules permettant d'augmenter les possibilités expressives de l'environnement, par exemple :

- un système de gestion avancée de calques pour permettre de dépasser la métaphore du carnet de croquis pour aboutir à celle, plus large, d'espace de dessin,

- Un module de reconnaissance de geste permettant grâce à une combinaison de l'interaction au stylo d'augmenter l'aspect naturel de l'environnement de travail.

En ce qui concerne l'interprétation de croquis, pour tenir compte de nos observations, nous proposons de faire évoluer EsQUiSE dans une direction relativement différente. Plutôt que de tenter de tout interpréter, nous sommes passés à un mode d'interaction explicite et déclaratif. Ainsi, au lieu de dessiner, il s'agit pour l'interprétation de surligner les éléments du dessin qui doivent être interprétés. Cette approche comprend deux avantages majeurs. D'une part, cet aspect déclaratif rend l'interprétation plus aisée : il y a moins d'ambiguïtés dues aux superpositions de traits et à leur imprécision. D'autre part, cela répond aux modes de travail «naturels» des concepteurs. La pratique du sur lignage est observée en papiercrayon pour expliciter le dessin. Cette modalité d'interaction a pour but de faire intervenir l'interprétation et le retour (modèle 3D) à des moments charnières, ponctuels et surtouts, décidés par le concepteur.

Enfin, à plus long terme, cette étude est un premier pas vers une interprétation d'esquisses utilisant la dynamique de tracé. En effet, plutôt que de tenter d'interpréter toutes les traces produites (brouillon et nettes), ou au contraire d'imposer à l'architecte d'expliciter son dessin, un logiciel d'interprétation pourrait analyser les dessins sur base du processus de mise au net. Les temps de réflexion, les tracés précis et lents, l'utilisation de la règle, sont autant d'indices d'opérations de mise au net. En approfondissant et en systématisant ces études de l'activité de transformation graphique, il sera peut-être possible de parvenir à un modèle dynamique de l'esquisse de conception, et d'interpréter les dessins sans contraindre ni interrompre le flux créatif de la conception architecturale.

\section{BIBLIOGRAPHIE}

Aliakseyeu, D., Martens, J.-B. and Rauterberg M. 2006. A computer support tool for the early stages of architectural design. Interacting with Computers, 18(4), 528-555.

Bae, S.-H., Balakrishnan, R. and Singh, K. 2008. ILoveSketch: As-natural-as-possible sketching system for creating 3D curve models. ACM Symposium on User Interface Software and Technology 2008, Monterey, CA, USA.

Bilda, Z. and Demirkan, H. 2003. An insight on designers' sketching activities in traditional versus digital media. Design Studies 24, 27-50. 
Brown, A.G.P. 2003. Visualization as a common design language: connecting art and science. Automation in Construction, 12, 703- 713.

Chen, X., Kang, S.B., Xu, Y.-Q., Dorsey, J. and Shum, H.-Y. 2008. Sketchingreality: Realistic interpretation of architectural designs. ACM Transations Graphics, 27(2), 1-15.

Coyette, A., Vanderdonckt, J. and Limbourg, Q. 2006. SketchiXML: A Design Tool for Informal User Interface Rapid Prototyping. Proceedings of RISE 2006, 160-176.

Goel V. 1995. Sketches of thought. Cambridge, MA, MIT Press,.

Gross, M. D. 1994. The Cocktail Napkin, the Fat Pencil, and the Slide Library. Proceedings of Association for Computer Aided Design in Architecture (ACADIA 94), St Louis, 103-113.

Huot S., Dumas C. and Hégron G. 2003. Toward Creative 3D Modeling: an Architects' Sketches Study. Actes de IFIP TC13 International Conference on Human-Computer Interaction (INTERACT'03), Zurich, Switzerland, 785-788.

Igarashi T., Matsuoka S. and Tanaka H. 1999. Teddy: A Sketching Interface for 3D Freeform Design. Proceedings of ACM SIGGRAPH'99, Los Angeles, 409-416.

Jatupoj, P. 2005. Sketchboard: The simple 3D modeling from architectural sketch recognition. Proceedings of Computer Aided Architectural Design in Asia (CAADIA 2005).

Juchmes, R., Leclercq, P., and Azar, S. 2004. A multi-Agent System for Architectural Sketches Interpretation. Proceedings of Eurographics Workshop on Sketch-Based interfaces and Modeling, Grenoble, France, 53-62.

Juchmes, R., Leclercq, P., and Azar, S. 2005. A feehand sketch environment for architectural design supported by a multi-agent system. Special issue of Computers and Graphics on Calligraphic Interfaces, 29(6).

Karpenko, O. A. and Hughes, J. F. 2006. Smoothsketch: 3D free-form shapes from complex sketches. Proceedings of ACM SIGGRAPH and ACM Trans. on Graphics, 25(3), 589598.

Kim, M.J. and Maher, M.L. 2008. The Impact of Tangible User Interfaces on Designers' Spatial Cognition. Human-Computer Interaction, 23(2), 101-137

Koutamanis, A. 2005. Sketching with Digital Pen and Paper. Proceedings of Computer Aided Architectural Design Futures 2005.

Kramer A., 1994. Translucent Patches - Dissolving Windows. Proceedings of ACM Symposium on User Interface Software and Technology, Marina del Rey, CA.

Landay, J. A. 1996. SILK: sketching interfaces like krazy. In M. J. Tauber (Ed.), CHI '96 Conference Companion on Human Factors in Computing Systems: Common Ground, 398- 399.

Lebahar, J.-C. 1983. Le dessin d'architecte. Simulation graphique et réduction d'incertitude. Editions Parenthèses, Paris. 
Leclercq, P. 2005. Le concept d'esquisse augmentée. Actes de SCAN'05 : Séminaire de Conception Architecturale Numérique, Paris, France.

Leclercq, P. and Elsen, C. 2007. Le croquis synthé-numérique. Actes de SCAN 07 : Séminaire de conception architecturale numérique, Liège, Belgique.

Lim , C.-K. (2003) Is a pen-based system just another pen or more than a pen? Proceedings of 21th eCAADe Conference.

MacCall, R., Ekaterini, V. and Zabel, J. 2001. Conceptual design as hypersketching. Proceedings of the 9th International Conference CAAD Futures, Kluwer Academic Publishers, Dordrecht, The Netherlands, 285-298.

Mayeur, A., Darses, F. and Leclercq, P. 2007. Apports de la visualisation de maquettes virtuelles 3D en phase d'esquisse architecturale. Actes d'Epique'07, Congrès de la Société Française de Psychologie

Neiman, B.,Do ; E. and Gross , M. 1999. Sketches and Their Functions in Early Design: A Retrospective Analysis of Two Houses. In W. Porter and G. Goldschmidt. (Eds.) Proceedings of Design Thinking Research Symposium '99.

Norman, D.A. 1998. The invisible computer, MIT Press, Cambridge University Press, MA.

Oh, J.-Y. Stuerzlinger, W. and Danahy, J. 2006. SESAME: towards better 3D conceptual design systems. Proceedings of the 6th conference on Designing Interactive systems.

Pranovich, S., Achten, H.H. and Wijk, J.J. 2002. Towards an Architectural Design System Based on Generic Representations. In Gero, J.S. (ed.). Artificial Intelligence in Design'02. Kluwer Academic Publishers, Dordrecht. 153-164.

Prost, R. 1992. Conception architecturale. Une investigation Méthodologique. L'Harmattan, Paris.

Rodgers, P.A., Green, G. and McGown A. 2000. Using concept sketches to track design progress. Design Studies, 21(5), 451-464.

Safin, S., Boulanger, C. and Leclercq, P. 2005. Premières évaluations d'un Bureau Virtuel pour un processus de conception augmenté. Actes de IHM'05: 17ème conférence francophone sur l'interaction homme-machine.

Safin, S., Delfosse, V. and Leclercq, P. 2010. Mixed-reality prototypes to support early creative design. In E. Dubois, P. Gray and L. Nigay (Eds). The Engineering of Mixed Reality Systems, London, Springer.

Safin, S., Leclercq, P. and Decortis, F. 2007. Impact d'un environnement d'esquisses virtuelles et d'un modèle 3D précoce surl'activité de conception architecturale. Revue d'Interaction Homme-Machine, 8(2), 65-98.

Shesh, A. and Chen, B. 2004. SMARTPAPER-An interactive and easy-to-use sketching system. Proceedings of Eurographics.

Van der Lugt, R. 2005. How sketching can affect the idea génération procès in design group meetings. Design Studies, 26(2), 101-122. 
Visser, W. (2006). The cognitive artifacts of designing. Lauwrence Erlbaum Associates.

Zeleznik, R.C., Herndon, K.P. and Hughes, J.F. 1996. SKETCH : an interface for sketching 3D scenes, Proceedings of SIGGRAPH'96, 163-170.

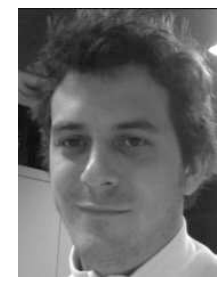

Stéphane Safin est chercheur à l'Université de Liège. Psychologue ergonome au laboratoire LUCID-ULg, il gère les aspects liés aux usages et l'intégration des utilisateurs finaux pour le développement de logiciels de modélisation et pour les dispositifs d'IHM à stylo numérique, principalement dans le domaine de l'architecture. Il mène aussi des recherches sur les activités de conception, sur le rôle des représentations externes dans le processus de conception créative en architecture, et sur les mécanismes de la collaboration en conception créative. Ces recherches sont orientées vers le développement d'outils innovants pour supporter la conception préliminaire.

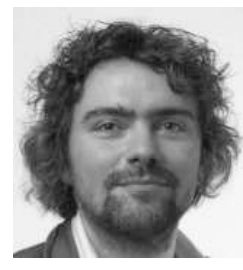

Roland Juchmes est professeur à l'Ecole Supérieure des Arts Saint-Luc de Liège et assistant au département Argenco de l'Université de Liège. Membre du laboratoire LUCID-ULg, ses travaux de recherche portent sur le développement de méthodes et d'outils d'assistance à la conception architecturale assistée par ordinateur (CAAO). Il s'intéresse particulièrement aux modèles et représentations de bâtiments adaptés aux phases préliminaires de conception.

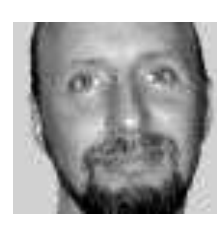

Pierre Leclercq est Ingénieur Civil Architecte et Docteur en Sciences de l'Ingénieur. Il est Professeur à la Faculté des Sciences Appliquées de l'Université de Liège (Belgique). Il y enseigne la méthodologie de projet, la modélisation assistée, l'analyse des processus de conception et les questions avancées de CAO. Il dirige le LUCID - Lab for User Cognition \& Innovative Design - équipe de recherche pluridisciplinaire à l'ULg. Il y mène recherches et développements en ingénierie de conception, associant les compétences de la modélisation virtuelle, de l'ergonomie cognitive, du génie logiciel et des interactions homme-machine avancées. 